\title{
Quantum indices and refined enumeration of real plane curves
}

\author{
by \\ Grigory MikHaLkin \\ Université de Genève \\ Carouge, Switzerland
}

\section{Introduction.}

\subsection{Quantum index}

Geometry of real algebraic curves in the plane is one of the most classical subjects in algebraic geometry.

It is easy to see that the logarithmic image $\log \left(\mathbb{R} C^{\circ}\right) \subset \mathbb{R}^{2}$ of any real algebraic curve $\mathbb{R} C^{\circ} \subset\left(\mathbb{R}^{\times}\right)^{2} \subset \mathbb{R}^{2}$ under the map

$$
\log (x, y)=(\log |x|, \log |y|)
$$

bounds a region of finite area in $\mathbb{R}^{2}$ (see Figures 3.1-3.3 for some examples of $\log \left(\mathbb{R} C^{\circ}\right.$ ) in degrees 1 and 2). Furthermore, this area is universally bounded from above for all curves of a given degree by the Passare-Rullgård inequality [25] for the area of amoebas.

E.g., if $\mathbb{R} C^{\circ}$ is a circle contained in the positive quadrant $\left(\mathbb{R}_{>0}\right)^{2}$, then it bounds a disk $D \subset\left(\mathbb{R}_{>0}\right)^{2}, \partial D=\mathbb{R} C^{\circ}$. The area of the disk $D$ is

$$
\int_{D} d x d y=\pi r^{2},
$$

where $r$ is its radius. Clearly, Area $(D)$ may be arbitrarily large. At the same time, it can be proved that the area of $\log D$ is

$$
\int_{D} \frac{d x}{x} \frac{d y}{y}<\pi^{2} .
$$

The inequality can be established either through direct computation or as a corollary of the Passare-Rullgård upper bound on the area of amoeba [25]. Thus, this logarithmic 
area of $D$ stays bounded no matter how large is the radius $r$. At the same time, it is clear that $\operatorname{Area}(\log (D))$ may assume any value between 0 and $\pi^{2}$.

In this paper, we impose the following conditions on an algebraic curve $\mathbb{R} C \subset \mathbb{R} \mathbb{P}^{2}$ (in the main body of the paper it is also formulated for other toric surfaces in place of $\mathbb{R P}^{2}$ ) so that such continuous behavior of the logarithmic area is no longer possible.

Namely, we assume that $\mathbb{R} C$ is an irreducible curve of type I (see $\S 2.1$ ). Then, according to [26], $\mathbb{R} C$ comes with a canonical orientation (defined up to simultaneous reversal in all components of $\mathbb{R} C$ ). This enables us to consider the signed area (with multiplicities) Area $\log (\mathbb{R} C)$ bounded by $\log \left(\mathbb{R} C^{\circ}\right) \subset \mathbb{R}^{2}$. Unless one of the two possible complex orientations of $\mathbb{R} C$ is chosen, $\operatorname{Area}_{\log }(\mathbb{R} C)$ is only well defined up to sign.

We have that the curve $\mathbb{R} C \subset \mathbb{R P}^{2}$ is the zero set of an irreducible homogeneous polynomial $f\left(x_{0}, x_{1}, x_{2}\right)$. For simplicity, in the introduction we assume that $\mathbb{R} C$ is disjoint from the points $(0: 0: 1),(0: 1: 0)$ and $(1: 0: 0)$. For $j=0,1,2$, the restriction of $f$ to the set $\left\{\left(x_{0}: x_{1}: x_{2}\right) \in \mathbb{R P}^{2}: x_{j}=0\right\}$ is a homogeneous polynomial $f_{j}$ in two variables responsible for the intersection of $\mathbb{R} C$ with the three coordinate axes of $\mathbb{R} \mathbb{P}^{2}$. We say that $\mathbb{R} C$ has real or purely imaginary coordinate intersection if for any (complex) zero $\left(z_{a}: z_{b}\right)$ of $f_{j}$ we have $\left(z_{b} / z_{a}\right)^{2} \in \mathbb{R}$. Theorem 3.1 asserts that in this case $\operatorname{Area}_{\log }(\mathbb{R} C)$ must be divisible by $\frac{1}{2} \pi^{2}$, and so cannot vary continuously. The number $k=\operatorname{Area}_{\log }(\mathbb{R} C) / \pi^{2}$ is thus a half-integer naturally associated with the curve. We call it the quantum index of $\mathbb{R} C$.

ThEOREM 3.1. (Special case of $\mathbb{R P}^{2}$ ) Let $\mathbb{R} C \subset \mathbb{R} \mathbb{P}^{2}$ be a real curve of degree $d$ and type I enhanced with a complex orientation. If $\mathbb{R} C$ has real or purely imaginary coordinate intersection, then

$$
\operatorname{Area}_{\log }(\mathbb{R} C)=k \pi^{2}
$$

with $k \in \frac{1}{2} \mathbb{Z}$ and $-\frac{1}{2} d^{2} \leqslant k \leqslant \frac{1}{2} d^{2}$.

To our knowledge, this classical-looking result is new even in the case $d=2$. Meanwhile the special case of $d=1$ is well known. The identity $\left|\operatorname{Area}_{\log }(\mathbb{R} C)\right|=\frac{1}{2} \pi^{2}$ in the case of lines was used by Mikael Passare [24] in his elegant new proof of Euler's formula $\zeta(2)=\frac{1}{6} \pi^{2}$. Another known special case of Theorem 3.1 is the case of the so-called simple Harnack curves introduced in [17]. As it was shown in [22], these curves have the maximal possible value of $\left|\operatorname{Area}_{\log }(\mathbb{R} C)\right|$ for their degree (equal to $\frac{1}{2} d^{2} \pi^{2}$ ). Simple Harnack curves have many geometric properties [17]. By now, these curves have appeared in a number of situations outside of real algebraic geometry, in particular in random perfect matchings of bipartite doubly periodic planar graphs of Richard Kenyon, Andrei Okounkov and Scott Sheffield [13]. The quantum index of Theorem 3.1 can be interpreted as a measure of proximity of a real curve to a simple Harnack curve.

Half-integrality of the quantum index $k$ may be explained through appearance of 
$2 k$ as the degree of some map as exhibited in Proposition 3.3. In accordance with this interpretation, Theorem 3.4 computes the quantum index through the degree of the real logarithmic Gauss map of $\mathbb{R} C$.

Theorem 4.7 studies the quantum index in the special case when $\mathbb{R} C$ is not only of type I, but also of toric type I (Definition 4.1). This condition implies that all coordinate intersections of $\mathbb{R} C$ are real. In this case, the quantum index may be refined to the index diagram (Definition 4.3), a closed broken lattice curve $\Sigma \subset \mathbb{R}^{2}$ well defined up to a translation by $2 \mathbb{Z}^{2}$.

The broken curve $\Sigma$ is an immersed multicomponent curve with each component corresponding to a component of the compactification $\mathbb{R} \bar{C}$ of $\mathbb{R} C^{\circ}$ defined by its Newton polygon $\Delta$. The complex orientation of $\mathbb{R} C$ induces an orientation of the closed broken curve $\Sigma$ so that we may compute the signed area Area $\Sigma$ inside $\Sigma$ which is a half-integer number, as the vertices of $\Sigma$ are integer.

THEOREM 4.7. (Simplified version) If $\mathbb{R} C \subset \mathbb{R P}^{2}$ is a real algebraic curve of toric type I enhanced with a choice of its complex orientation, then its quantum index $k$ coincides with Area $\Sigma$.

Each edge of $\Sigma$ corresponds to an intersection of $\mathbb{R} \bar{C}$ with a toric divisor of the toric variety $\mathbb{R} \Delta$ corresponding to the Newton polygon $\Delta$, and thus to a side $E \subset \partial \Delta$. If this intersection is transversal, then the corresponding oriented edge of $\Sigma$ is given by the primitive integer outer normal vector $\vec{n}(E)$. More generally, it is given by $\vec{n}(E)$ times the multiplicity of the intersection. This makes finding the index diagram $\Sigma$, and thus the quantum index $k$, extremely easy at least in the case of rational curves with real coordinate intersection (cf., e.g., Figures 3.2 and 4.1).

The index diagram $\Sigma$ can be viewed as a non-commutative version of the Newton polygon $\Delta$ : it is made from the same elements (the vectors $\vec{n}(E)$ taken $\#\left(E \cap \mathbb{Z}^{2}\right)-1$ times) as $\partial \Delta$, but the real structure on $\mathbb{R} \bar{C}$ gives those pieces a cyclic order (in the case of connected $\mathbb{R} \bar{C}$ ), or divides these elements into several cyclically ordered subsets.

Recall that Mikael Forsberg, Mikael Passare and August Tsikh in [7] have defined the amoeba-index map, which is a locally constant map on the complement $\mathbb{R}^{2} \backslash \mathcal{A}$ of the amoeba $\mathcal{A}=\log \left(\mathbb{C} C^{\circ}\right)$ of the complexification $\mathbb{C} C^{\circ}$ of $\mathbb{R} C^{\circ}$. With each connected component of $\mathbb{R}^{2} \backslash \mathcal{A}$ is associated a lattice point of the Newton polygon $\Delta$.

For toric-type-I curves the formula (4.4) defines the real-index map so that each connected component of the normalization $\mathbb{R} \widetilde{C}^{\circ}$, or a solitary real singularity of $\mathbb{R} C^{\circ}$, acquires a real index which is a lattice point of the convex hull of the index diagram $\Sigma$. Theorem 4.16 computes the amoeba-index map in terms of the linking number with the curve $\mathbb{R} C^{\circ}$ enhanced with the real indices. 


\subsection{Refined real enumerative geometry in the plane}

The second part of the paper is devoted to applications of the quantum index of real curves introduced in this paper to enumerative geometry over complex and real numbers. The space of planar projective rational curves of degree $d$ is $(3 d-1)$-dimensional. Thus, given a generic configuration $\mathcal{P}$ of $3 d-1$ points in the projective plane, we expect a finite set $\mathcal{S}_{d}$ of such curves. What we can do next with this set depends on our choice of ground field.

Our two main choices are the fields $\mathbb{C}$ and $\mathbb{R}$ of complex and real numbers. For both of these cases we choose $\mathcal{P} \subset \mathbb{R} \mathbb{P}^{2}$ generically and denote with $\mathcal{S}_{d}^{\mathbb{C}}$ (resp. $\mathcal{S}_{d}^{\mathbb{R}}$ ) the finite set of all planar projective rational curves of degree $d$ defined over $\mathbb{C}$ (resp. over $\mathbb{R}$ ) passing through $\mathcal{P}$. It is easy to see that the cardinality $N_{d}^{\mathbb{C}}=\# \mathcal{S}_{d}^{\mathbb{C}}$ does not depend on the choice of $\mathcal{P}$ (even if $\mathcal{P}$ is chosen generically in $\mathbb{C P}^{2}$ rather than in $\mathbb{R} \mathbb{P}^{2}$ ). At the same time, the cardinality $\# \mathcal{S}_{d}^{\mathbb{R}}$ depends on the choice of the generic configuration $\mathcal{P}$ and a priori only the parity of this set remains invariant.

According to the seminal result of Welschinger [28], the curves $\mathbb{R} C \in \mathcal{S}_{d}^{\mathbb{R}}$ come with natural signs $w(\mathbb{R} C)= \pm 1$, so that the integer number

$$
N_{d}^{\mathbb{R}}=\sum_{\mathbb{R} C \in \mathcal{S}_{d}^{\mathbb{R}}} w(\mathbb{R} C)
$$

is independent of the choice of $\mathcal{P}$. The number $N_{d}^{\mathbb{R}}$ is thus known as the Welschinger invariant and is a fundamental notion of real enumerative geometry. Itenberg, Kharlamov and Shustin in [10] have established non-trivial lower bounds on $\# \mathcal{S}_{d}^{\mathbb{R}}$ with the help of $N_{d}^{\mathbb{R}}$.

Both integer numbers $N_{d}^{\mathbb{C}}$ and $N_{d}^{\mathbb{R}}$ were simultaneously computed with the help of passing to the tropical limit in [18]. Namely, $N_{d}^{\mathbb{C}}$ and $N_{d}^{\mathbb{R}}$ can be presented as sums of multiplicities of corresponding tropical curves passing through a generic configuration of points in the tropical plane. The tropical curves are the same in both cases, however the rules for defining their $\mathbb{C}$ and $\mathbb{R}$ multiplicities are different, so the sums $N_{d}^{\mathbb{C}}$ and $N_{d}^{\mathbb{R}}$ are different as well.

With the help of this presentation, Block and Göttsche in [1] have proposed combining the numbers $N_{d}^{\mathbb{C}}$ and $N_{d}^{\mathbb{R}}$ into a single number $N_{d}^{\text {trop }}$, which is no longer an integer number, but an integer $q$-number (a Laurent polynomial in $q$ with positive integer coefficients invariant under the substitution $q \mapsto 1 / q)$. The value at $q=1$ is capable to recover the number of complex curves, while the value at $q=-1$ should be capable to recover the number of real curves in the same enumerative problem. E.g., there are $q+10+q^{-1}$ many of rational cubic curves passing through eight generic points in $\mathbb{R} \mathbb{P}^{2}$. At the same 
time, there are twelve curves over $\mathbb{C}$ and eight curves over $\mathbb{R}$ (if we count real curves with the Welschinger sign [28]).

Conjecturally (see [8]), the $q$-refinement $N_{d}^{\text {trop }}$ of the integer number $N_{d}^{\mathbb{C}}$ agrees with the $\chi_{y}$-genus refinement of Severi degrees proposed by Göttsche and Shende in [8]. Also this refinement looks to be at least vaguely resemblant of the refinements of DonaldsonThomas invariants considered by Kontsevich and Soibelman [15] and by Nekrasov and Okounkov [23] in some other frameworks (in particular, for 3-folds).

The quantum index allows us to obtain a refined enumeration of planar curves entirely within classical real algebraic geometry of the plane with the help of Theorem 5.5. Once again, for simplicity, we discuss only the special case of the projective plane here in the introduction, while in the main body of the paper the theorem is formulated for other toric surfaces as well.

Recall that the space of rational curves of degree $d$ in $\mathbb{C P}^{2}$ is $(3 d-1)$-dimensional. Thus, we expect a finite number of such curves if we impose on them $3 d-1$ conditions. Let us choose a generic configuration of $3 d-1$ points on the three coordinate axes of $\mathbb{C P}^{2}$ (the $x$-axis, the $y$-axis and the $\infty$-axis), so that each axis contains no more than $d$ points: e.g., there are $d$ generic points on the $x$ - and $y$-axes and $3 d-1$ generic points on the $\infty$-axis. The elementary generalization of the classical Menelaus theorem (see Figure 6.2) found already by Carnot [4] (later further generalized as the Weil reciprocity law) ensures that there is a unique $3 d$-th point on the $\infty$-axis such that any irreducible curve of degree $d$ passing through our $3 d-1$ points also passes through the $3 d$-th point. The resulting configuration of $3 d$ points on the union of three coordinate axes varies in a $(3 d-1)$-dimensional family of Menelaus configurations.

We define the square map Sq: $\mathbb{C P}^{2} \rightarrow \mathbb{C P}^{2}$ by $\operatorname{Sq}\left(z_{0}: z_{1}: z_{2}\right)=\left(z_{0}^{2}: z_{1}^{2}: z_{2}^{2}\right)$. Consider a configuration $\mathcal{P}$ of $3 d$ points on the coordinate axes of $\mathbb{R P}^{2}$ such that there exists a Menelaus configuration $\mathcal{Q}$ on the coordinate axes of $\mathbb{C P}^{2}$ with $\mathcal{P}=\operatorname{Sq}(\mathcal{Q})$. A point of $\mathcal{Q}$ is either real or purely imaginary (depending on the signs of the coordinates of its image under Sq). An irreducible rational curve $\mathbb{R} C \subset \mathbb{R} \mathbb{P}^{2}$ such that $\mathrm{Sq}(\mathbb{C} C)$ passes through $\mathcal{P}=\operatorname{Sq}(\mathcal{Q})$ is of type I and has real or purely imaginary coordinate intersection. Thus, the quantum index $k$ is well defined.

In (5.5) we define $R_{d, k}(\mathcal{P})$ (here we write $d$ instead of $\Delta$ since we restrict ourselves to the special case of $\mathbb{R P}^{2}$ in the introduction) as one quarter of the number of irreducible oriented rational curves $\mathbb{R} C \subset \mathbb{R} \mathbb{P}^{2}$ of degree $d$ and quantum index $k$ such that $\mathrm{Sq}(\mathbb{C} C)$ passes through $\mathcal{P}$. Each curve $\mathbb{R} C$ here is taken with the sign (5.3), which is a modification of the Welschinger sign [28]. Note that such curves come in quadruples due to the action of the deck transformations of the four-to-one covering Sq $\left.\right|_{\left(\mathbb{R}^{\times}\right)^{2}}:\left(\mathbb{R}^{\times}\right)^{2} \rightarrow\left(\mathbb{R}^{\times}\right)^{2}$. This is the reason for including $\frac{1}{4}$ in the definition of $R_{d, k}(\mathcal{P})$. The points of $\mathcal{P}$ contained 
in the closure of the positive quadrant $\left(\mathbb{R}_{>0}\right)^{2}$ (positive points) correspond to the real coordinate axes intersections of $\mathbb{R} C$; the other (negative) points correspond to the purely imaginary coordinate axes intersections.

The image of each component of $\mathbb{C} C \backslash \mathbb{R} C$ under Sq may be viewed as an open holomorphic disk $F$ in $\mathbb{C P}^{2}$ with the boundary contained in the closure $L=\overline{\left(\mathbb{R}_{>0}\right)^{2}}$ of the positive quadrant. The subspace $L \subset \mathbb{C P}^{2}$ is a Lagrangian submanifold with boundary. The positive points of $\mathcal{P}$ correspond to the tangencies of $\partial F$ and $\partial L$, while the negative points of $\mathcal{P}$ correspond to the intersections of the open disk $F$ with the coordinate axes of $\mathbb{R} \mathbb{P}^{2}$ away from $\partial L$. From this viewpoint, $R_{d, k}(\mathcal{P})$ is the number of certain holomorphic disks whose boundary is contained in $L$, a framework widely used in symplectic geometry. An unconventional feature is the presence of the boundary in the contractible (and thus orientable) Lagrangian surface $L$. The positive points of $\mathcal{P}$ are contained in the boundary $\partial L$. The holomorphic disks are tangent to $\partial L$ at these points. The negative points of $\mathcal{P}$ are disjoint from $L$, and thus from the boundaries of the holomorphic disks.

The number of negative points on the three coordinate axes is given by a triple $\lambda=\left(\lambda_{1}, \lambda_{2}, \lambda_{3}\right)$ with $\lambda_{j} \leqslant d$.

Theorem 5.5. (Special case of $\mathbb{R} \mathbb{P}^{2}$ ) Suppose that $\mathcal{P}=\mathrm{Sq}(\mathcal{Q})$ for a generic Menelaus configuration $\mathcal{Q}$ of $3 d$ points on the coordinate axes of $\mathbb{C P}^{2}$. If $\mathcal{P} \subset \mathbb{R} \mathbb{P}^{2}$, then the number $R_{d, k, \lambda}=R_{d, k}(\mathcal{P})$ is well defined. It is independent of the choice of $\mathcal{P}$ and depends only on $d, k$ and $\lambda$.

In particular, $R_{d, k}(\mathcal{P})$ depends only on $d$ and $k$ when all points of $\mathcal{P}$ are positive, i.e. when the Menelaus configuration $\mathcal{Q}$ is real itself.

For a positive point $p \in \mathcal{P}$ the inverse image $\mathrm{Sq}^{-1}(p)$ consists of two points: a positive one $p_{+} \in \partial L$ and a negative one $p_{-} \notin \partial L$. The condition $\mathrm{Sq}(\mathbb{C} C) \ni p$ is equivalent to the condition that $\mathbb{R} C$ passes through $p_{+}$or $p_{-}$. Note that the invariance claimed in Theorem 5.5 relies on including into $R_{d, k}(\mathcal{P})$ both of these possibilities. If we leave out only the curves passing through $p_{+}$(or $p_{-}$) as in (5.6), then the resulting sum $\widetilde{R}_{d, k}(\mathcal{P})$ is no longer invariant under deformations of $\mathcal{P}$. Nevertheless, a partial invariance result for $\widetilde{R}_{d, k}(\mathcal{P})$ is provided by Theorem 5.7.

The generating function $R_{d}(\lambda)=\sum_{k} R_{d, k, \lambda} q^{k}$ defined in (5.9) is a Laurent polynomial

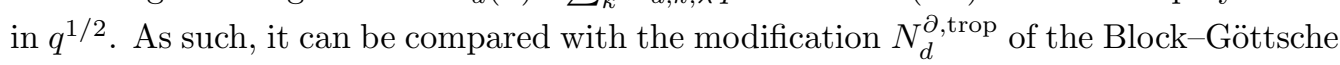
refined tropical invariants $N_{d}^{\text {trop }}$, where we take for $\mathcal{P}$ a generic Menelaus configuration of points in the boundary $\partial \mathbb{T} \mathbb{P}^{2}=\mathbb{P}^{2} \backslash \mathbb{R}^{2}$, rather than a generic configuration of points

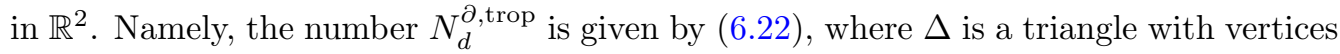
$(0,0),(d, 0)$ and $(0, d)$. The last theorem of the paper is an identity between $R_{d}=$ $R_{d}(0,0,0)$ and $N_{d}^{\partial \text {,trop }}$. 
TheOREM 5.9. (Special case of $\mathbb{R P}^{2}$ )

$$
R_{d}=\left(q^{1 / 2}-q^{-1 / 2}\right)^{3 d-2} N_{d}^{\partial, \text { trop }}
$$

This theorem has a surprising corollary, due to the coincidence of the number $N_{d}^{\partial, \mathbb{C}}$ of irreducible rational complex curves $\mathbb{C} C \subset \mathbb{C P}^{2}$ of degree $d$ passing through $\mathcal{P}$ with the value $N_{d}^{\partial \text {,trop }}(1)$. Thus, both of these numbers are completely determined by $R_{d}$, the number accounting only for curves defined over $\mathbb{R}$. Note that for this purpose it is crucial to use the quantum refinement by $q^{k}$, since for $q=1$ we would have to divide by zero (the value of $\left(q^{1 / 2}-q^{-1 / 2}\right)^{3 d-2}$ at $\left.q^{1 / 2}=1\right)$ to recover $N_{d}^{\partial \text {,trop }}(1)$.

\section{Conventions and notation}

\subsection{Real curves of type I and their complex orientation}

A real curve $\mathbb{R} C \subset \mathbb{R} \mathbb{P}^{2}$ is given by a single homogeneous polynomial equation

$$
F\left(z_{0}, z_{1}, z_{2}\right)=\sum_{j+k+l=d} a_{k, l} z_{0}^{j} z_{1}^{k} z_{2}^{l}=0, \quad a_{k, l} \in \mathbb{R} .
$$

The locus $\mathbb{C} C \subset \mathbb{C P}^{2}$ of complex solutions of $F=0$ is called the complexification of $\mathbb{R} C$. We assume $F$ to be irreducible over $\mathbb{C}$ and such that $\mathbb{C} C$ does not coincide with a coordinate axis $\left\{\left(z_{0}: z_{1}: z_{2}\right) \in \mathbb{C P}^{2}: z_{j}=0\right\}, j=0,1,2$. The normalization

$$
\nu: \mathbb{C} \widetilde{C} \longrightarrow \mathbb{C} C
$$

defines a parametrization of $\mathbb{C} C$ by a Riemann surface $\mathbb{C} \widetilde{C}$. The antiholomorphic involution of complex conjugation conj acts on $\mathbb{C} C$ in an orientation-reversing way, so that its fixed point locus is $\mathbb{R} C$. The restriction of conj to the smooth locus of $\mathbb{C} C$ lifts to an antiholomorphic involution conj: $\mathbb{C} \widetilde{C} \rightarrow \mathbb{C} \widetilde{C}$ on the normalization. We denote the fixed point locus of $\widetilde{\text { conj }}$ by $\mathbb{R} \widetilde{C}$. Clearly, $\nu(\mathbb{R} \widetilde{C}) \subset \mathbb{R} C$. Irreducibility of $\mathbb{C} C$ is equivalent to connectedness of $\mathbb{C} \widetilde{C}$.

Following Felix Klein, we say that $\mathbb{R} C$ is of type I if $\mathbb{C} \widetilde{C} \backslash \mathbb{R} \widetilde{C}$ is disconnected. In such case it consists of two connected components $S$ and $S^{\prime}=\widetilde{\operatorname{conj}}(S)$, which are naturally oriented by the complex orientation of the Riemann surface $\mathbb{C} \widetilde{C}$. We have $\mathbb{R} \widetilde{C}=\partial S=\partial S^{\prime}$, so a choice of one of these components, say $S$, induces the boundary orientation on $\mathbb{R} \widetilde{C}$. The resulting orientation is called a complex orientation of $\mathbb{R} \widetilde{C}$ and is subject to Rokhlin's complex orientation formula [26]. If we choose $S^{\prime}$ instead of $S$, then the orientations of all components of $\mathbb{R} C$ will reverse simultaneously. Thus, any orientation of a component of $\mathbb{R} \widetilde{C}$ determines a component of $\mathbb{C} \widetilde{C} \backslash \mathbb{R} \widetilde{C}$. 


\subsection{Toric viewpoint and reality of coordinate intersections}

The projective plane $\mathbb{C P}^{2}$ can be thought of as the toric compactificationof the torus $\left(\mathbb{C}^{\times}\right)^{2}$. The curve $\mathbb{C} C \subset \mathbb{C P}^{2}$ is the closure of its toric part $\mathbb{C} C^{\circ}=\mathbb{C} C \cap\left(\mathbb{C}^{\times}\right)^{2}$. The complement $\partial \mathbb{C P}^{2}=\mathbb{C P}^{2} \backslash\left(\mathbb{C}^{\times}\right)^{2}$ is the union of three axes: the $x$-axis, the $y$-axis and the $\infty$-axis. These axes intersect pairwise at the points $(1: 0: 0),(0: 1: 0),(0: 0: 1) \in \mathbb{R} \mathbb{P}^{2}$. If the coefficients $a_{0,0}, a_{d, 0}$ and $a_{0, d}$ are non-zero, then $\mathbb{C} C$ is disjoint from the intersection points of the axes. In the general case, it is reasonable to consider other toric surfaces compactifying $\left(\mathbb{C}^{\times}\right)^{2}$, so that the closure of $\mathbb{C} C^{\circ}$ is disjoint from pairwise intersections of toric divisors.

Let us consider the (non-homogeneous) polynomial $f(x, y)=F(1, x, y)$ and its Newton polygon

$$
\Delta=\operatorname{Conv}\left\{(j, k) \in \mathbb{R}^{2}: a_{j, k} \neq 0\right\},
$$

where Conv denotes the convex hull. If $\Delta$ has non-empty interior, then the dual fan to $\Delta$ defines a toric compactification $\mathbb{C} \Delta \supset\left(\mathbb{C}^{\times}\right)^{2}$. The toric divisors of $\mathbb{C} \Delta$ correspond to the sides of $\Delta$. Their pairwise intersections correspond to the vertices of $\Delta$ and are disjoint from the compactification $\mathbb{C} \bar{C}$ of the curve $\mathbb{C} C^{\circ}$. We denote the union of toric divisors by $\partial \mathbb{C} \Delta \subset \mathbb{C} \Delta$. Accordingly, we denote the real part of $\mathbb{C} \Delta$ (resp. $\partial \mathbb{C} \Delta, \mathbb{C} C^{\circ}, \mathbb{C} \bar{C}$ ) by $\mathbb{R} \Delta$ (resp. $\partial \mathbb{R} \Delta, \mathbb{R} C^{\circ}, \mathbb{R} \bar{C}$ ). For example, we have $\mathbb{R} \mathbb{P}^{2}=\mathbb{R} \Delta$, with $\Delta$ being the triangle $\operatorname{Conv}\{(0,0),(1,0),(0,1)\}$ or a positive integer multiple of it.

Let Sq: $\left(\mathbb{C}^{\times}\right)^{2} \rightarrow\left(\mathbb{C}^{\times}\right)^{2}$ be the map defined by $\operatorname{Sq}(x, y)=\left(x^{2}, y^{2}\right)$. This map extends to a map $\mathrm{Sq}^{\Delta}: \mathbb{C} \Delta \rightarrow \mathbb{C} \Delta$.

We call a point $p \in \mathbb{C} \Delta$ real or purely imaginary if $\mathrm{Sq}^{\Delta}(p) \in \mathbb{R} \Delta$. We say that a curve $\mathbb{R} C \subset \mathbb{R} \mathbb{P}^{2}$ has real or purely imaginary coordinate intersection if every point of $\mathbb{C} C \cap \partial \mathbb{C} \Delta$ is real or purely imaginary.

\subsection{Logarithmic area and other numbers associated with a type-I real curve}

Let $\mathbb{R} C$ be a real curve of type I enhanced with a choice of a complex orientation. Consider the image $\log \left(\mathbb{R} C^{\circ}\right) \subset \mathbb{R}^{2}$, where $\log :\left(\mathbb{C}^{\times}\right)^{2} \rightarrow \mathbb{R}^{2}$ is the map defined in (1.1). For a point $p \in \mathbb{R}^{2} \backslash \log \left(\mathbb{R} C^{\circ}\right)$ we define ind $(p) \in \mathbb{Z}$ to be the intersection number of an oriented ray $R \subset \mathbb{R}^{2}$ emanating from $x$ in a generic direction and the oriented curve $\log \left(\mathbb{R} C^{\circ}\right)$ (this number can be considered as the linking number of $p$ and $\log \left(\mathbb{R} C^{\circ}\right)$ ).

Definition 2.1. The integral

$$
\operatorname{Area}_{\log }(\mathbb{R} C)=\int_{\mathbb{R}^{2}} \operatorname{ind}_{\mathbb{R} C}(x) d x
$$

is called the logarithmic area of $\mathbb{R} C$. 
This is the signed area encompassed by $\log \left(\mathbb{R} C^{\circ}\right)$, where the area of each region of $\mathbb{R}^{2} \backslash \log \left(\mathbb{R} C^{\circ}\right)$ is taken with the multiplicity equal to the linking number of $\log \left(\mathbb{R} C^{\circ}\right)$.

Let $S \subset \mathbb{C} \widetilde{C} \backslash \mathbb{R} \widetilde{C}$ be the component corresponding to the chosen complex orientation of $\mathbb{R} \widetilde{C}$. The intersection points $\nu(S) \cap\left(\mathbb{R}^{\times}\right)^{2}$ are the so-called solitary real singularities of $\mathbb{R} C^{\circ}$. The multiplicity of a solitary real singularity $p \in \nu(S) \cap\left(\mathbb{R}^{\times}\right)^{2}$ is the local intersection number of $S$ and $\left(\mathbb{R}^{\times}\right)^{2}$ at $p$. Here the orientation of $S$ is induced by the inclusion $S \subset \mathbb{C} \widetilde{C}$, while the orientation of $\left(\mathbb{R}^{\times}\right)^{2}$ is induced by the covering $\left.\log \right|_{\left(\mathbb{R}^{\times}\right)^{2}}:\left(\mathbb{R}^{\times}\right)^{2} \rightarrow \mathbb{R}^{2}$. In other words, the quadrants $\mathbb{R}_{>0}^{2}$ and $\mathbb{R}_{<0}^{2}$ are counterclockwise oriented, while the quadrants $\mathbb{R}_{>0} \times \mathbb{R}_{<0}$ and $\mathbb{R}_{<0} \times \mathbb{R}_{>0}$ are clockwise oriented. The toric solitary singularities number $E(\mathbb{R} C) \in \mathbb{Z}$ is the sum of the multiplicities over all solitary real singularities of $\mathbb{R} C^{\circ}$, i.e. the total intersection number of $S$ and $\left(\mathbb{R}^{\times}\right)^{2}$ (enhanced with our choice of orientation).

The logarithmic Gauss map sends a smooth point of $\mathbb{R} C^{\circ}$ to the tangent direction of the corresponding point on $\log (\mathbb{R} C) \subset \mathbb{R}^{2}$. This map uniquely extends to a map

$$
\gamma: \mathbb{R} \widetilde{C} \longrightarrow \mathbb{R P}^{1}
$$

cf. [12], [17]. The logarithmic rotation number $\operatorname{Rot}_{\log }(\mathbb{R} C) \in \mathbb{Z}$ is the degree of $\gamma$.

\section{Quantum indices of real curves.}

ThEOREM 3.1. Let $\mathbb{R} C \subset \mathbb{R} \mathbb{P}^{2}$ be a real curve of type $I$ enhanced with a complex orientation. If $\mathbb{R} C$ has real or purely imaginary coordinate intersection, then

$$
\operatorname{Area}_{\log }(\mathbb{R} C)=k \pi^{2}
$$

where $k \in \frac{1}{2} \mathbb{Z}$,

$$
-\operatorname{Area}(\Delta) \leqslant k \leqslant \operatorname{Area}(\Delta)
$$

and $k \equiv \operatorname{Area}(\Delta)(\bmod 1)$.

Note that, as $\Delta \subset \mathbb{R}^{2}$ is a lattice polynomial, its area is a half-integer number.

Definition 3.2. We say that $k(\mathbb{R} C)=\operatorname{Area}_{\log }(\mathbb{R} C) / \pi^{2}$ is the quantum index of $\mathbb{R} C$.

If $\mathbb{R} C$ is an irreducible real curve of type I with real or purely imaginary coordinate intersection, but the complex orientation of $\mathbb{R} C$ is not fixed, then its quantum index is well defined up to sign.

The quantum index $k(\mathbb{R} C)$ can also be expressed without computing the logarithmic area. 
Proposition 3.3. The integer number $2 k(\mathbb{R} C)$ coincides with the degree of the map

$$
\text { 2Arg: } \mathbb{C} C^{\circ} \backslash \mathbb{R} C^{\circ} \longrightarrow(\mathbb{R} / \pi \mathbb{Z})^{2}
$$

i.e. with the number of inverse images at a generic point of the torus $(\mathbb{R} / \pi \mathbb{Z})^{2}$ counted with the sign according to the orientation. (In particular, this number does not depend on the choice of a point in $(\mathbb{R} / \pi \mathbb{Z})^{2}$ as long as this choice is generic.) Here the orientation of $\mathbb{C} C^{\circ} \backslash \mathbb{R} C^{\circ}$ is defined by the condition that it coincides with the complex orientation of $\mathbb{C} C$ on the component $S \subset \mathbb{C} C^{\circ} \backslash \mathbb{R} C^{\circ}$ determined by the orientation of $\mathbb{R} C$ and is opposite to the complex orientation of $\mathbb{C} C$ on the component conj $(S) \subset \mathbb{C} C^{\circ} \backslash \mathbb{R} C^{\circ}$. The map $2 \operatorname{Arg}$ is defined by $2 \operatorname{Arg}(x, y)=(2 \arg (x), 2 \arg (y))$.

We say that $\mathbb{R} \bar{C}$ is transversal to $\partial \mathbb{R} \Delta$ if for any $p \in \mathbb{R} C \cap \partial \mathbb{R} \Delta$ we have $\nu^{-1}(p) \subset \mathbb{R} \widetilde{C}$, the composition $\mathbb{R} \widetilde{C} \rightarrow \mathbb{R} \widetilde{C} \subset \mathbb{R} \Delta$ is an immersion near $\nu^{-1}(p) \subset \mathbb{R} \widetilde{C}$, and this immersion is transversal to $\partial \mathbb{R} \Delta$.

THEOREM 3.4. Let $\mathbb{R} C$ be a curve of type I with real or purely imaginary coordinate intersection such that $\mathbb{R} \bar{C}$ is transversal to $\partial \mathbb{R} \Delta$. Then

$$
k(\mathbb{R} C)=-\frac{1}{2} \operatorname{Rot}_{\log }(\mathbb{R} C)+E(\mathbb{R} C) .
$$

If $\mathbb{R} \bar{C}$ is not transversal to $\partial \mathbb{R} \Delta$, then an adjustment of the right-hand side according to the order of tangency and the orientation of $\mathbb{R} C$ should be added to the formula of Theorem 3.4.

Example 3.5. (Simple Harnack curves) If $\mathbb{R} C^{\circ} \subset\left(\mathbb{R}^{\times}\right)^{2}$ is a simple Harnack curve (see [17]), then $k(\mathbb{R} C)= \pm \operatorname{Area}(\Delta)$. Vice versa, if $k(\mathbb{R} C)= \pm \operatorname{Area}(\Delta)$, then $\mathbb{R} C^{\circ}$ is a simple Harnack curve; see [22]. This characterizes real curves of the highest and lowest quantum index.

Example 3.6. (Quantum indices of real lines) Any real line is a curve of type I and has real coordinate intersection. The quantum index of a real line in $\mathbb{R} \mathbb{P}^{2}$ disjoint from the points (1:0:0), (0:1:0) and (0:0:1) is $\pm \frac{1}{2}$ (depending on its orientation); see Figure 3.1. The quantum index of a line passing through exactly one of these points is zero.

Example 3.7. (Quantum indices of real conics) A smooth non-empty real conic is a curve of type I. Figure 3.2 depicts real conics in $\mathbb{R P}^{2}$ that intersect the coordinates axes in six real points.

Note that a circle in $\mathbb{R}^{2}$ intersects the infinite axis of $\mathbb{R P}^{2}$ at the points $(0: 1: \pm i)$. Thus, a circle intersecting the coordinate axes of $\mathbb{R}^{2}$ in four real points has real or purely imaginary coordinate intersection; see Figure 3.3. A circle passing through the origin in $\mathbb{R}^{2}$ has quantum index $\pm \frac{1}{2}$. Otherwise, the quantum index of a circle is \pm 1 or zero. 

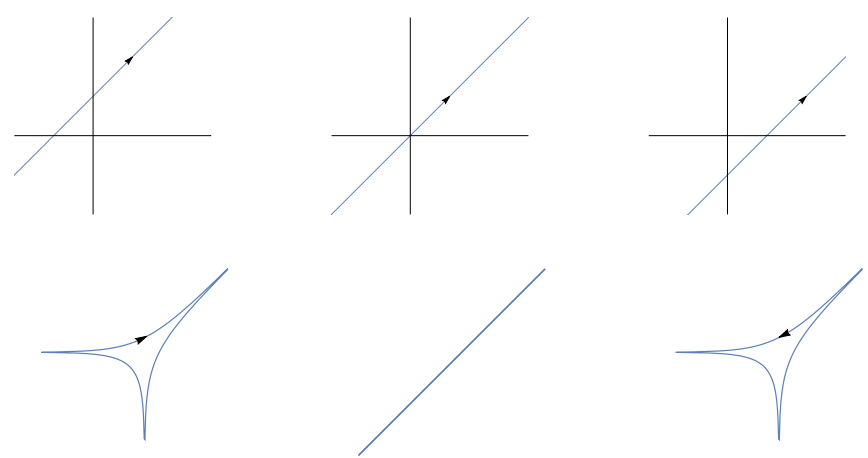

$k=-1$

$k=0$

$k=1$

Figure 3.1. Oriented lines, their logarithmic images and quantum indices.

\section{Toric-type-I curves: quantum indices and diagrams}

\subsection{Definition of toric-type-I curves and their index diagrams}

Denote by $\mathbb{C} \widetilde{C}^{\circ} \subset \mathbb{C} \widetilde{C}$ the normalization of an algebraic curve $\mathbb{C} C^{\circ} \subset\left(\mathbb{C}^{\times}\right)^{2}$ and by $\mathbb{R} \widetilde{C}^{\circ}$ its real part. The composition of the normalization and the inclusion map induces a map $\mathbb{C} \widetilde{C}^{\circ} \backslash \mathbb{R} \widetilde{C}^{\circ} \rightarrow\left(\mathbb{C}^{\times}\right)^{2}$.

Definition 4.1. We say that an irreducible real algebraic curve $\mathbb{R} C^{\circ} \subset\left(\mathbb{R}^{\times}\right)^{2}$ has toric type $\mathrm{I}$ if $\mathbb{R} C$ is of type I (see $\S 2.1$ ) and the induced homomorphism

$$
H_{1}\left(\mathbb{C} \widetilde{C}^{\circ} \backslash \mathbb{R} \widetilde{C}^{\circ}\right) \longrightarrow H_{1}\left(\left(\mathbb{C}^{\times}\right)^{2}\right)=\mathbb{Z}^{2}
$$

is trivial.

Each side $E \subset \Delta$ is dual to a unique primitive integer vector $\vec{n}(E) \subset \mathbb{Z}^{2}$ (which sits in the space dual to the vector space containing the Newton polygon $\Delta$ ) oriented away from $\Delta$. We refer to $\vec{n}(E)$ as the normal vector to $E \subset \partial \Delta$.

Proposition 4.2. If $\mathbb{R} C^{\circ} \subset\left(\mathbb{R}^{\times}\right)^{2}$ is of toric type $\mathrm{I}$, then

$$
\mathbb{C} \bar{C} \cap \partial \mathbb{C} \Delta \subset \mathbb{R} \bar{C} \subset \mathbb{R} \Delta \text {. }
$$

In other words, $\mathbb{R} C$ has real coordinate intersection. Thus, it has a well-defined quantum index for any of its two complex orientations.

Proof. The homology class in $H_{1}\left(\left(\mathbb{C}^{\times}\right)^{2}\right)=\mathbb{Z}^{2}$ of a small loop in $\mathbb{C} C^{\circ}$ around a point of $\mathbb{C} \bar{C} \cap \partial \mathbb{C} \Delta$ is a positive multiple of $\vec{n}(E)$ for a side $E \subset \Delta$. Therefore, this class is non-zero. Thus, such a loop must intersect $\mathbb{R} C^{\circ}$ if $\mathbb{R} C^{\circ}$ is of toric type $\mathrm{I}$. 



$k= \pm 2$

$k= \pm 1$

$k=0$

$k=\mp 1$

Figure 3.2. Projective hyperbolas, their logarithmic images and quantum indices.

Definition 4.3. A continuous map $a: \Sigma \rightarrow \mathbb{R}^{2}$ from a graph $\Sigma$ is called the index diagram of the curve $\mathbb{R} C^{\circ}$ of toric type I enhanced with a choice of the complex orientation corresponding to $S \subset \mathbb{C} \widetilde{C} \backslash \mathbb{R} \widetilde{C}$, if the following conditions hold.

- The vertices of the graph $\Sigma$ are parameterized by the connected components $K^{\circ} \subset$ $\mathbb{R} \widetilde{C}^{\circ}$. We denote the vertex corresponding to $K^{\circ}$ by $v\left(K^{\circ}\right) \in \Sigma$.

- The image $a\left(v\left(K^{\circ}\right)\right)=(a, b) \in \mathbb{Z}^{2}$ is a lattice point in $\mathbb{R}^{2}$ such that $K^{\circ}$ is contained in the $\left((-1)^{a},(-1)^{b}\right)$-quadrant of $\left(\mathbb{R}^{\times}\right)^{2}$.

- Vertices $v\left(K_{1}^{\circ}\right), v\left(K_{2}^{\circ}\right) \in \Sigma$ are connected with an oriented edge $e$ (which we identify with the straight oriented interval $[0,1])$ if and only if $K_{1}^{\circ}$ and $K_{2}^{\circ}$ are adjacent to a point $p_{e} \in \mathbb{R} \widetilde{C}$ in the order defined by the complex orientation of $\mathbb{R} \widetilde{C}$. (Clearly, both $K_{1}^{\circ}$ and $K_{2}^{\circ}$ are non-compact in such case.)

- The restriction $\left.a\right|_{e}: e \approx[0,1] \rightarrow \mathbb{R}^{2}$ is an affine map with

$$
a\left(v\left(K_{2}^{\circ}\right)\right)-a\left(v\left(K_{1}^{\circ}\right)\right)=m_{e} \vec{n}(E) .
$$

Here, $m_{e}$ is the local intersection number of $\mathbb{C} \widetilde{C}$ and $\partial \mathbb{C} \Delta$ at $p_{e}$.

- There exists a continuous map

$$
\tilde{l}: \widetilde{S}=(S \backslash \partial \mathbb{C} \Delta) \cup \mathbb{R} \widetilde{C}^{\circ} \longrightarrow \mathbb{C}^{2}
$$

holomorphic on $\widetilde{S} \backslash \mathbb{R} \widetilde{C}^{\circ}$ such that $e^{\pi \tilde{l}}$ coincides with the tautological map $\widetilde{S} \rightarrow\left(\mathbb{C}^{\times}\right)^{2}$, while for every connected component $K^{\circ} \subset \mathbb{R} \widetilde{C}^{\circ}$ we have

$$
\operatorname{Im} \tilde{l}\left(K^{\circ}\right)=a\left(v\left(K^{\circ}\right)\right) .
$$



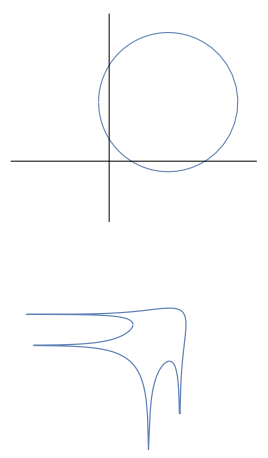

$k= \pm 1$
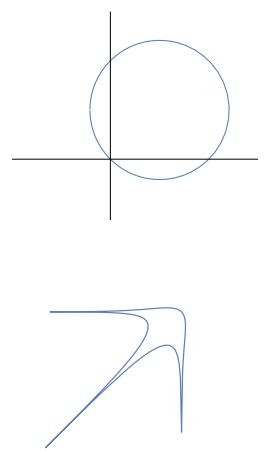

$k= \pm \frac{1}{2}$
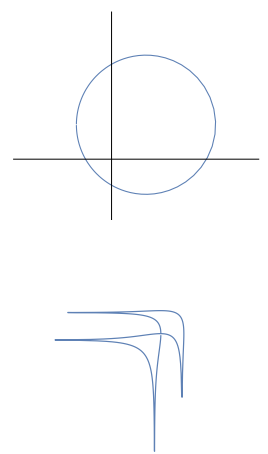

$k=0$

Figure 3.3. Circles, their logarithmic images and quantum indices.

Here, both the exponent $e^{\pi \tilde{l}}$ and the imaginary part $\operatorname{Im} \tilde{l}\left(K^{\circ}\right)$ are understood coordinatewise.

Topologically, the graph $\Sigma$ is the disjoint union of $n$ circles and $m$ points, where $n$ is the number of components of $\mathbb{R} \widetilde{C}$ intersecting $\partial \mathbb{R} \Delta$, and $m$ is the number of compact components of $\mathbb{R} \widetilde{C}^{\circ}$.

Denote by $\bar{\Sigma} \subset \mathbb{R}^{2}$ the convex hull of $a(\Sigma)$. The map

$$
\alpha: K^{\circ} \longmapsto \operatorname{Im} \tilde{l}\left(K^{0}\right)=a\left(v\left(K^{\circ}\right)\right) \in \bar{\Sigma} \cap \mathbb{Z}^{2}
$$

defined on the components of $\mathbb{R} \widetilde{C}^{\circ}$ is called the real-index map. Since the map $\tilde{l}$ is holomorphic, its imaginary part $\operatorname{Im} \tilde{l}$ is harmonic, and thus $\operatorname{Im} \tilde{l}(\widetilde{S}) \subset \bar{\Sigma}$.

Proposition 4.4. Any curve $\mathbb{R} C^{\circ} \subset\left(\mathbb{R}^{\times}\right)^{2}$ of toric type I admits an index diagram $\Sigma(\mathbb{R} C) \subset \mathbb{R}^{2}$ which is unique up to a translation by $2 \mathbb{Z}^{2}$ in $\mathbb{R}^{2}$.

Proof. Since $\mathbb{R} C$ is of toric type I, the surface $\widetilde{S} \subset\left(\mathbb{C}^{\times}\right)^{2}$ lifts under the exponent map $\mathbb{C}^{2} \rightarrow\left(\mathbb{C}^{\times}\right)^{2}$. Translating the lift by integer multiples of $\pi$, if needed, ensures that $(a, b)+2 i \mathbb{Z}^{2} \subset \mathbb{C}^{2}$ corresponds to the lift of the $\left((-1)^{a},(-1)^{b}\right)$-quadrant in $\left(\mathbb{C}^{\times}\right)^{2}$. Denote this lift by $\tilde{l}$, and define the map $a$ on the vertices of $\Sigma$ by (4.4). An edge $e \subset \Sigma$ is mapped to the image of the accumulation set at the end of $\widetilde{S}$ corresponding to $e$. To check the condition (4.2), we change coordinates in $\left(\mathbb{C}^{\times}\right)^{2}$ multiplicatively, so that the toric divisor corresponding to $e$ is the $x$-axis. Then $\tilde{l}$ maps the accumulation set at the $e$-end of $\widetilde{S}$ to the vertical interval of length $2 m_{e}$. Reversing the coordinate change, we recover an interval parallel to $\vec{n}(E)$.

Note that for each connected component $K \subset \mathbb{R} \widetilde{C}$ (which is necessarily closed) with $K \cap \partial \mathbb{R} \Delta \neq \varnothing$ formula (4.2) already determines the part $a(K): \Sigma(K) \rightarrow \mathbb{R}^{2}$ corresponding 
to $K$ of the index diagram $a: \Sigma \rightarrow \mathbb{R}^{2}$, up to a translation in $\mathbb{R}^{2}$. Indeed, it suffices to choose arbitrarily $\alpha\left(K^{\circ}\right)$ for an $\operatorname{arc} K^{\circ} \subset K \backslash \partial \mathbb{R} \Delta$, and proceed inductively.

Proposition 4.5. If $\mathbb{R} C^{\circ}$ is a curve of toric type $\mathrm{I}$, then the broken line a $(\Sigma(K))$ resulting from inductive application of (4.2) is closed for any connected component $K$ of $\mathbb{R} \widetilde{C}$ with $K \cap \partial \mathbb{R} \Delta \neq \varnothing$.

Conversely, suppose that $\mathbb{R} \widetilde{C}$ is an $M$-curve (i.e. the number of its components is one plus the genus of $\mathbb{C} \widetilde{C})$ with $(\mathbb{C} \widetilde{C} \backslash \mathbb{R} \widetilde{C}) \cap \partial \mathbb{C} \Delta=\varnothing$ such that the broken line defined inductively by (4.2) for every connected components $K \subset \mathbb{R} \widetilde{C}$ is closed. Then, $\mathbb{R} C^{\circ}$ has toric type $I$.

Proof. The first part of the statement is a corollary of Proposition 4.4. Conversely, for an $M$-curve $\mathbb{R} \widetilde{C}$, each component of $\mathbb{C} \widetilde{C} \backslash \mathbb{R} \widetilde{C}$ is a sphere with punctures corresponding to the components of $\mathbb{R} \widetilde{C}$. The homology class of a loop, for each component, is determined inductively by (4.2). It is zero, by our hypothesis.

\subsection{Quantum index and toric complex orientation formula}

Let $\mathbb{R} C^{\circ} \subset\left(\mathbb{R}^{\times}\right)^{2}$ be a curve of toric type I enhanced with the complex orientation corresponding to a component $S \subset \mathbb{C} \widetilde{C} \backslash \mathbb{R} \widetilde{C}$. Denote by

$$
\text { Area } \Sigma \in \frac{1}{2} \mathbb{Z}
$$

the signed area (with multiplicities) enclosed by $a(\Sigma)$ in $\mathbb{R}^{2}$.

Let $p \in \mathbb{R}^{2}$ be a point, let $R_{\varepsilon} \subset \mathbb{R}^{2}$ be the oriented ray emanating from $p$ in a generic direction $\varepsilon$ in $\mathbb{R}^{2}$. Define $\operatorname{lk}_{\varepsilon}(p, \Sigma)$ to be the intersection number of the image $a(\Sigma)$ and $R_{\varepsilon}$ in points other than $p$. If $p \notin a(\Sigma)$, then this number is the index of $p$ with respect to $a(\Sigma)$ (considered in $\S 2.3$ ), and does not depend on the choice of $\varepsilon$. Otherwise, $\operatorname{lk}_{\varepsilon}(p, \Sigma)$ depends on $\varepsilon$.

For each quadrant $Q=\left((-1)^{a},(-1)^{b}\right) \mathbb{R}_{>0}^{2}$, we define

$$
\operatorname{lk}_{\varepsilon}(Q, \Sigma)=\sum_{k_{a}, k_{b} \in \mathbb{Z}} \mathrm{lk}_{\varepsilon}\left(\left(a+2 k_{a}, b+2 k_{b}\right), \Sigma\right) \in \mathbb{Z} .
$$

Any connected component $K \subset \mathbb{R} \widetilde{C}$ disjoint from $\partial \mathbb{R} \Delta$ is contained in a single quadrant $Q$. The image $\log (K)$ is a closed oriented curve in $\mathbb{R}^{2}$. Let $\lambda(K) \in \mathbb{Z}$ be the rotation number of $\log (K)$, i.e. the degree of the logarithmic Gauss map of $K \subset \mathbb{R}^{2}$. E.g., if $K \subset \mathbb{R}^{2}$ is a positively oriented embedded circle contained in the $(+,+)$ - or $(-,-)$-quadrant (resp. in the $(+,-)$ - or $(-,+)$-quadrant), then $\lambda(K)=1$ (resp. $\lambda(K)=-1)$. Any point of $S \cap Q$ is a real isolated singular point $p \in \mathbb{R} C^{\circ}$. We denote by $\lambda(p) \in \mathbb{Z}$ the intersection number 
of $S$ and $Q$. Recall that the orientation of $\left(\mathbb{R}^{\times}\right)^{2}$ (and thus, of $Q$ ) is defined in $\S 2.3$ as the pull-back of the standard orientation of $\mathbb{R}^{2}$ by $\left.\left.\log \right|_{Q}\right)$.

If $K^{\circ} \subset \mathbb{R} \widetilde{C}^{\circ}$ is a connected component (not necessarily compact), then the local degree $\lambda_{\varepsilon}\left(K^{\circ}\right)$ of the oriented logarithmic Gauss map $\left.\widetilde{\gamma}\right|_{K^{\circ}}: K^{\circ} \rightarrow \widetilde{\mathbb{R P}}^{1}$ at a point $\varepsilon \in \widetilde{\mathbb{R P}}^{1}$ may depend on the choice of $\varepsilon$. For $(a, b) \in \mathbb{Z}^{2}$ and $\varepsilon \in \widetilde{\mathbb{R P}}^{1} \backslash \widetilde{\mathbb{Q P}^{1}}$, we set

$$
\lambda_{\varepsilon}(a, b)=-\sum_{K^{\circ}} \lambda_{\varepsilon}\left(K^{\circ}\right)+\sum_{p} \lambda(p),
$$

where the sums are taken over all components $K^{\circ} \subset \mathbb{R} \widetilde{C}^{\circ}$ with $\alpha\left(K^{\circ}\right)=(a, b)$ and all isolated singular points $p$ of $\mathbb{R} C^{\circ}$ with $\operatorname{Im} \tilde{l}(p)=(a, b)$. The following statement is straightforward (with the help of the maximum principle for $\operatorname{Im} \tilde{l}$ ).

Proposition 4.6. The number $\lambda_{\varepsilon}(a, b)$ does not depend on $\varepsilon$ if $(a, b) \notin a(\Sigma)$. If $(a, b) \notin \bar{\Sigma}$, then $\lambda_{\varepsilon}(a, b)=0$.

For each quadrant $Q=\left((-1)^{a},(-1)^{b}\right) \mathbb{R}_{>0}^{2} \subset\left(\mathbb{R}^{\times}\right)^{2}$, we may take the sum

$$
\lambda_{\varepsilon}(Q)=\sum_{k_{a}, k_{b} \in \mathbb{Z}} \lambda_{\varepsilon}\left(a+2 k_{a}, b+2 k_{b}\right) .
$$

The result is independent on the translation ambiguity in the definition of the real-index map.

THEOREM 4.7. If $\mathbb{R} C^{\circ} \subset\left(\mathbb{R}^{\times}\right)^{2}$ is a real algebraic curve of toric type I enhanced with a choice of its complex orientation, then

$$
k(\mathbb{R} C)=\operatorname{Area} \Sigma(\mathbb{R} C) .
$$

For each $(a, b) \in \mathbb{Z}^{2}$ and $\varepsilon \in \widetilde{\mathbb{R P}}^{1} \backslash \widetilde{\mathbb{Q P}}^{1}$ we have

$$
\lambda_{\varepsilon}(a, b)=\mathrm{lk}_{\varepsilon}((a, b), \Sigma) .
$$

COROLlary 4.8. For a curve of toric type I with index diagram $\Sigma$, we have

$$
\lambda_{\varepsilon}(Q)=\mathrm{lk}_{\varepsilon}(Q, \Sigma)
$$

for each quadrant $Q \subset\left(\mathbb{R}^{\times}\right)^{2}$.

Equality (4.11) may be viewed as the toric complex orientation formula for torictype-I curves.

COROLlary 4.9. The total number of closed components of a curve $\mathbb{R} \widetilde{C}^{\circ}$ of toric type $I$ and its solitary real singularities is not less than the number of lattice points $(a, b) \in \mathbb{Z}^{2} \backslash \Sigma$ with $\operatorname{lk}((a, b), \Sigma) \neq 0$. 

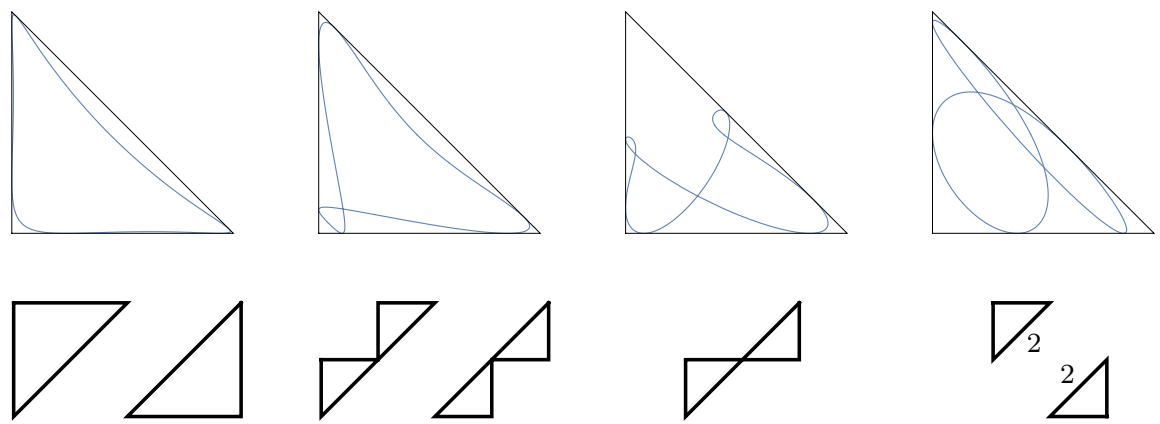

Figure 4.1. Squares of the real conics from Figure 3.2 and their diagrams $\Sigma$ for both possible orientations.

Proof. If $\operatorname{lk}((a, b), \Sigma) \neq 0$ then, by (4.10), $\lambda(a, b) \neq 0$ and thus there exists a closed component or a solitary real singularity of $\mathbb{R} C^{\circ}$ of real index $(a, b)$.

Example 4.10. All real rational curves which intersect $\partial \mathbb{R} \Delta$ in $\#\left(\partial \Delta \cap \mathbb{Z}^{2}\right)$ points (counted with multiplicity) have toric type I, as $\mathbb{C} \widetilde{C}^{\circ} \backslash \mathbb{R} \widetilde{C}^{\circ}$ is the disjoint union of two open disks. Therefore, we may compute the quantum index of such curves with the help of Theorem 4.7 .

Figure 4.1 depicts the images of the real conics from Figure 3.2 under $\mathrm{Sq}^{\Delta}$ (reparameterized with the help of the moment map). Each of these conics may be oriented in two different ways producing two different diagrams. For one of these conics the diagrams for the two opposite orientations coincide. For the other three conics they are different.

Note that, in the case where $\mathbb{C} \Delta=\mathbb{C P}^{2}$, the orientation can be uniquely recovered from the diagram, as the edges correspond to the normals to $\Delta$. E.g. the vertical edges are always directed downwards.

Remark 4.11. The diagram $\Sigma$ may be viewed as a non-commutative version of the polygon $\Delta$. Here, the set of normal vectors is given a cyclic order.

Note that

$$
- \text { Area } \Delta \leqslant \text { Area } \Sigma \leqslant \text { Area } \Delta
$$

for any (possibly multicomponent) closed broken curve $\Sigma$ whose oriented edges are normal vectors to $\Delta$, so that each side $E \subset \Delta$ contribute to $\#\left(E \cap \mathbb{Z}^{2}\right)-1$ normal vectors (counted with multiplicity). Furthermore, we have Area $\Sigma= \pm$ Area $\Delta$ if and only if $\Sigma \subset \mathbb{R}^{2}$ is a single-component broken curve coinciding with the polygon $\Delta$ itself rotated by 90 degrees (as we can represent the primitive normal vector to a vector $(a, b) \in \mathbb{Z}^{2}$ by $(-b, a)$, identifying the vector space $\mathbb{R}^{2}$ with its dual).

Recall the notion of cyclically maximal position of $\mathbb{R} \bar{C} \subset \mathbb{R} \Delta$ in $\mathbb{R} \Delta$ from $[17$, Definition 2]. It can be rephrased that $\mathbb{R} \bar{C}$ has a connected component $K$ intersecting $\partial \mathbb{R} \Delta$ in 
$m=\#\left(\partial \Delta \cap \mathbb{Z}^{2}\right)$ points counted with multiplicity, and the cyclic order of the intersection points on $K$ agrees with the cyclic order of the corresponding normal vectors to $\partial \Delta$. This condition is equivalent to equality (4.12). It was proved in [17] that, for each $\Delta$, the topological type of the triad $\left(\mathbb{R} \Delta ; \mathbb{R} \bar{C}_{\Delta}, \partial \mathbb{R} \Delta\right)$ is unique if a curve $\mathbb{R} \bar{C}$ with Newton polygon $\Delta$ has cyclically maximal position and is smooth and transversal to $\partial \mathbb{R} \Delta$. In this case, $\left(\mathbb{R} \Delta ; \mathbb{R} \bar{C}_{\Delta}, \partial \mathbb{R} \Delta\right)$ is called the simple Harnack $\Delta$-triad.

The number of points in $(\Delta \backslash \partial \Delta) \cap \mathbb{Z}^{2}$ is equal to the arithmetic genus $g$ of $\mathbb{R} \bar{C}$. On the other hand, Corollary 4.9 implies that the total number of closed connected components of $\mathbb{R} C^{\circ}$ and its isolated real singular points is at least $g$. Thus, all closed components of $\mathbb{R} C^{\circ}$ are smooth ovals, all singular points of $\mathbb{R} C^{\circ}$ are solitary double points, and the curve $\mathbb{C} C^{\circ}$ is a nodal $M$-curve. We get the following statement.

Corollary 4.12. If $\mathbb{R} C^{\circ} \subset\left(\mathbb{R}^{\times}\right)^{2}$ is a curve of toric type I with

$$
\text { Area } \Sigma(\mathbb{R} C)= \pm \text { Area } \Delta
$$

then it is an $M$-curve whose only singularities are solitary real nodes.

Furthermore, the topological type of $(\mathbb{R} \Delta ; \mathbb{R} \bar{C}, \partial \mathbb{R} \Delta)$ is obtained from the simple Harnack $\Delta$-triad $\left(\mathbb{R} \Delta ; \mathbb{R} \bar{C}_{\Delta}, \partial \mathbb{R} \Delta\right)$ by contracting some of the ovals of $\mathbb{R} C_{\Delta}^{\circ}$ to solitary double points and replacing some $n$-tuples of consecutive transversal intersection points of $\mathbb{R} \bar{C}_{\Delta}$ and $\partial \mathbb{R} \Delta$ (sitting on the same toric divisor) with points of $n$-th order of tangency.

Proof. The curve $\mathrm{Sq}^{\Delta}\left(\mathbb{C} C^{\circ}\right)$ also has toric type I. Its diagram is obtained from $\Sigma(\mathbb{R} C)$ by scaling both coordinates by 2 , so the quantum index of $\mathrm{Sq}^{\Delta}\left(\mathbb{C} C^{\circ}\right)$ is equal to \pm Area $(2 \Delta)$. Corollary 4.9 implies that the only singularities of $\mathrm{Sq}^{\Delta}\left(\mathbb{C} C^{\circ}\right)$ are solitary real nodes, so that $\mathrm{Sq}^{\Delta}(\mathbb{R} \bar{C})$ does not have self-intersections. Therefore, for each toric divisor $\mathbb{R} E$, the order of intersection points on $\mathbb{R} E$ and that on the component $K \subset \mathbb{R} \widetilde{C}$ agree.

Let us look at the compact components of $\mathbb{R} C^{\circ}$. Their number and distribution among the quadrants of $\left(\mathbb{R}^{\times}\right)^{2}$ is determined by the lattice points of $\Sigma(\mathbb{R} C)$, and thus by $\Delta$. Furthermore, Corollary 4.9 implies that in each quadrant of $\left(\mathbb{R}^{\times}\right)^{2}$ all ovals and solitary real nodes of $\mathbb{R} C^{\circ}$ have the same orientation. The complex orientation formula [26] ensures that these components cannot be nested among themselves, and that they are arranged with respect to $K$ so that their complex orientation is coherent with the complex orientation of $K$.

Remark 4.13. The proof of Corollary 4.9 is applicable also for pseudoholomorphic, and even the so-called flexible (see [27]) real curves of toric type I. Thus, Corollary 4.12 may be considered as a further generalization of the topological uniqueness theorem for simple Harnack curves [17], from its version for pseudoholomorphic curves [2] recently found by Erwan Brugallé. 


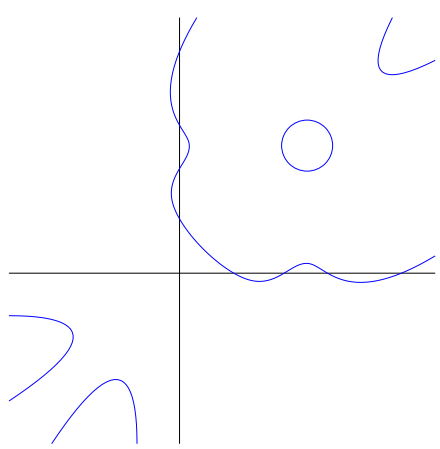

Figure 4.2. A quartic curve of type I, but not of toric type I.

Example 4.14. The curve sketched on Figure 4.2 is isotopic to a smooth real quartic curve of type I. Namely, it can be obtained as a perturbation of the union of four lines. However, it is not an $M$-curve, while its diagram coincides with that of the simple Harnack curve of the same degree (i.e. the triangle with vertices $(0,0),(0,-4)$ and $(-4,-4)$, for one of the orientations). By Corollary 4.12, this curve is not of toric type I. In other words, there is a cycle in $\mathbb{C} C^{\circ} \backslash \mathbb{R} C^{\circ}$ that is homologically non-trivial in $H_{1}\left(\left(\mathbb{C}^{\times}\right)^{2}\right)$. Also, we can deduce this from the toric complex orientation formula (4.11).

\subsection{The real-index map vs. the amoeba-index map}

To advance the viewpoint of the index diagram $\Sigma$ as a non-commutative version of the Newton polygon $\Delta$, it is interesting to compare the real-index map (4.4) for toric-type-I curves and the amoeba-index map

$$
\text { ind: } \mathbb{R}^{2} \backslash \log \left(\mathbb{C} C^{\circ}\right) \longrightarrow \Delta \cap \mathbb{Z}^{2}
$$

of Forsberg-Passare-Tsikh [7]. The map (4.13) is locally constant, and thus it indexes the components $K$ of the amoeba complement $\mathbb{R}^{2} \backslash \log \left(\mathbb{C} C^{\circ}\right)$ by lattice points of $\Delta$.

One obvious distinction between ind and $\alpha$ is that they take values in dual spaces: the Newton polygon $\Delta$ belongs to the dual vector space to $\mathbb{R}^{2}=\log \left(\mathbb{C}^{\times}\right)^{2}$. But, due to the symplectic form $\omega((a, b),(c, d))=a d-b c, a, b, c, d \in \mathbb{R}$, we have a preferred isomorphism between these spaces. Denote by $(a, b)^{*}=(b,-a)$ the corresponding identification.

As usual, we fix a complex orientation on $\mathbb{R} \widetilde{C}$ and consider the corresponding component $S^{\circ} \subset \mathbb{C} \widetilde{C}^{\circ} \backslash \mathbb{R} \widetilde{C}^{\circ}$. Let

$$
p, p^{\prime} \in \mathbb{R}^{2} \backslash \log \left(\mathbb{C} C^{\circ}\right)
$$


and let $\gamma \subset \mathbb{R}^{2}$ be a smooth path between $p$ and $p^{\prime}$. We assume $\gamma$ to be in general position with respect to $\mathbb{R} C^{\circ}$. The intersection number $\#(\gamma, \log \mathbb{R} C) \in \mathbb{Z}$ is well defined, as $\log (\mathbb{R} C) \subset \mathbb{R}^{2}$ is proper.

Proposition 4.15. We have $\#(\gamma, \log \mathbb{R} C)=0$.

Proof. The local degree of the map $\left.\log \right|_{S^{\circ}}: S^{\circ} \rightarrow \mathbb{R}^{2}$ changes along $\gamma$ according to the intersection with $\mathbb{R} C^{\circ}$. Since the local degree at the endpoints of $\gamma$ vanishes, we have $\#(\gamma, \log \mathbb{R} C)=0$.

Using the real-index map (4.4), we may refine the intersection number

$$
\#(\gamma, \log \mathbb{R} C)=0
$$

to

$$
\#_{\alpha}\left(\gamma, \log \mathbb{R} C^{\circ}\right)=\sum_{q \in \log ^{-1}(\gamma) \cap \mathbb{R} C^{\circ}} \#_{q}\left(\gamma, \log \mathbb{R} C^{\circ}\right) \alpha(q) \in \mathbb{Z}^{2} .
$$

Here, $\#_{q}\left(\gamma, \log \mathbb{R} C^{\circ}\right)= \pm 1$ is the local intersection number between $\gamma$, and $\log \mathbb{R} C^{\circ}$ and $\alpha(q) \in \mathbb{Z}^{2}$ is the real index of the component of $\mathbb{R} \widetilde{C}^{\circ}$ containing the point $q$.

THEOREM 4.16. Let $\mathbb{R} C^{\circ} \subset\left(\mathbb{R}^{\times}\right)^{2}$ be an algebraic curve of toric type I. For any two points $p, p^{\prime} \in \mathbb{R}^{2} \backslash S^{\circ} \cup \mathbb{R} C^{\circ}$ and a generic smooth path $\gamma \subset \mathbb{R}^{2}$ connecting $p$ and $p^{\prime}$, we have

$$
\left(\operatorname{ind}\left(p^{\prime}\right)-\operatorname{ind}(p)\right)^{*}=\#_{\alpha}\left(\gamma, \log \mathbb{R} C^{\circ}\right)
$$

Proof. Consider the 1-dimensional submanifold

$$
M=\left(\left.\log \right|_{\mathbb{C} C^{\circ}}\right)^{-1}(\gamma) \subset \mathbb{C} C^{\circ}
$$

Its orientation is induced by that of $\gamma$ through the pull-back map, with the help of the orientations of the ambient spaces: the standard orientation $\mathbb{R}^{2} \supset \gamma$ and the complex orientation of $\mathbb{C} C^{\circ} \supset M$. Since $\gamma$ is chosen generically, the 1-submanifold $M$ is smooth.

Any component of $M$ disjoint from $\mathbb{R} C^{\circ}$ is null-homologous in $\left(\mathbb{C}^{\times}\right)^{2}$, as $\mathbb{R} C^{\circ}$ is a toric-type-I curve. A component $L \subset M$ intersecting $\mathbb{R} C^{\circ}$ consists of two arcs interchanged by conj. Let $q \in \mathbb{R} C^{\circ}$ be the source and $q^{\prime} \in \mathbb{R} C^{\circ}$ be the target of the arc $\delta=L \cap S^{\circ}$ with the orientation induced from $M$. We have

$$
\operatorname{Im} \tilde{l}\left(q^{\prime}\right)-\operatorname{Im} \tilde{l}(q)=\alpha\left(q^{\prime}\right)-\alpha(q)
$$

by (4.4), and therefore $[L]=\alpha\left(q^{\prime}\right)-\alpha(q) \in \mathbb{Z}^{2}=H_{1}\left(\left(\mathbb{C}^{\times}\right)^{2}\right)$ so that $[M] \in H_{1}\left(\left(\mathbb{C}^{\times}\right)^{2}\right)$ is given by the right-hand side of (4.15). 
By [17], we may interpret ind $(p)$ as the linear functional on

$$
H_{1}\left(\log ^{-1}(p)\right)=\mathbb{Z}^{2}
$$

that associates with each oriented loop $N \subset \log ^{-1}(p)$ the linking number of $N$ and the closure of the surface $\mathbb{C} C^{\circ}$ in $\mathbb{C}^{2}$. Suppose that $N^{\prime} \subset \log ^{-1}\left(p^{\prime}\right)$ is a loop homologous to $N$ in $\left(\mathbb{C}^{\times}\right)^{2}$, so that $N^{\prime}-N=\partial P$ for a surface $P \subset\left(\mathbb{C}^{\times}\right)^{2}$. Then, the difference of the linking numbers of $N^{\prime}$ and $N$ coincides with the intersection number of $P$ and $\mathbb{C} C^{\circ}$. Choosing the membrane $P$ to be contained in $\log ^{-1}(\gamma)$, we identify the difference with the intersection number of $[N]$ and $[M]$ in

$$
\mathbb{Z}^{2}=H_{1}\left(\log ^{-1}(p)\right)=H_{1}\left(\left(\mathbb{C}^{\times}\right)^{2}\right)=H_{1}\left(S^{1} \times S^{1}\right) .
$$

\section{Refined real enumerative geometry}

\subsection{Invariance of real refined enumeration}

Let $\Delta \subset \mathbb{R}^{2}$ be a lattice polygon with non-empty interior. Let $E_{j} \subset \partial \Delta, j=1, \ldots, n$, be its sides of integer length $m_{j}=\#\left(E_{j} \cap \mathbb{Z}^{2}\right)-1$, enumerated counterclockwise. We denote by $v_{j} \in \Delta$ the vertices of $\Delta$ enumerated so that $E_{j}$ connects $v_{j-1}$ and $v_{j}$ (using the convention $\left.v_{0}=v_{n}\right)$. Let $\mathbb{C} E_{j}$ be the corresponding toric divisor. Let $\mathcal{P}=\left\{p_{l}\right\}_{l=1}^{m}$ be a collection of $m=\#\left(\partial \Delta \cap \mathbb{Z}^{2}\right)$ points on $\partial \mathbb{C} \Delta$. We do not assume the points $p_{l}$ to be distinct, but assume that exactly $m_{j}$ of these points are contained in the toric divisor $\mathbb{C} E_{j}$ (in particular, we have $\mathcal{P} \cap \bigcup_{j=1}^{n}\left\{v_{j}\right\}=\varnothing$ ).

The primitive vector $\left(a_{j}, b_{j}\right) \in \mathbb{Z}^{2}$ parallel to $E_{j}$ and coherent with the counterclockwise orientation of $\partial \Delta$ defines the multiplicative-linear (monomial) map

$$
\begin{aligned}
\pi_{j}:\left(\mathbb{C}^{\times}\right)^{2} & \longrightarrow \mathbb{C}^{\times}, \\
(z, w) & \longmapsto z^{a_{j}} w^{b_{j}} .
\end{aligned}
$$

This map extends to a continuous map $\bar{\pi}_{j}:\left(\mathbb{C}^{\times}\right)^{2} \cup\left(\mathbb{C} E_{j} \backslash\left\{v_{j-1}, v_{j}\right\}\right)$. Define the map

$$
\varrho: \bigcup_{j=1}^{n} \mathbb{C} E_{j} \backslash\left\{0_{j}, \infty_{j}\right\} \longrightarrow \mathbb{C}^{\times}
$$

by $\varrho(p)=\bar{\pi}_{j}(p)$ for $p \in \mathbb{C} E_{j}$.

Remark 5.1. Note that the two coordinates $z$ and $w$ in $\left(\mathbb{C}^{\times}\right)^{2}$ give two meromorphic functions on the Riemann surface $\mathbb{C} \widetilde{C}$ obtained through normalization $\bar{\nu}: \mathbb{C} \widetilde{C} \rightarrow \mathbb{C} \bar{C}$. Le symbole modéré (defined by J. Tate according to [5])

$$
(z, w)_{\tilde{p}}=(-1)^{v(z) v(w)}\left[w^{v(z)} z^{-v(w)}\right](\tilde{p}) \in \mathbb{C}^{\times}
$$


for a point $\tilde{p} \in \mathbb{C} \widetilde{C}$ is determined, up to the sign, by its image $p=\bar{\nu}(\tilde{p}) \in \mathbb{C} \Delta$. The notation $v(z)$ refers to the valuation of $z$ at $p$, i.e. to the order of zero (or pole with the negative sign) of this meromorphic function. The square brackets $\left[w^{v(z)} z^{-v(w)}\right](\tilde{p})$ refer to taking the limit of the function $w^{v(z)} z^{-v(w)}$ when the argument tends to $\tilde{p}$. Note that the result is always a non-zero complex number. Equivalently, we can write

$$
(z, w)_{\tilde{p}}= \begin{cases}1, & \text { if } p \in\left(\mathbb{C}^{\times}\right)^{2}, \\ (-1)^{v(z) v(w)} \varrho^{-a}(p), & \text { if } p \in \partial \mathbb{C} \Delta,\end{cases}
$$

where $a$ is the order of tangency of $\mathbb{C} \widetilde{C}$ to $\partial \mathbb{C} \Delta$ at $\tilde{p}$.

Condition 5.2. (Menelaus condition on $\mathcal{P}$ )

$$
\prod_{l=1}^{m} \varrho\left(p_{l}\right)=(-1)^{m}
$$

The following proposition is known as the Menelaus theorem in the case where $\mathbb{R} \Delta=\mathbb{R} \mathbb{P}^{2}$ and $m=3$ (lines), and generalized by Carnot [3] to higher-degree curves. It is also known as the Weil reciprocity law, see e.g. [9].

Proposition 5.3. (Cf. [5, formula (1.2)]) There exists a curve $\mathbb{C} \bar{C} \subset \mathbb{C} \Delta$ such that

$$
\mathbb{C} \bar{C} \cap \partial \mathbb{C} \Delta=\mathcal{P}
$$

(in the sense that each point $p \in \mathbb{C} \bar{C} \cap \partial \mathbb{C} \Delta$ is included in $\mathcal{P}$ a number of times equal to the local intersection number of $\mathbb{C} \bar{C}$ and $\partial \mathbb{C} \Delta$ at $p$ ) if and only if (5.2) holds.

Proof. The torus part $\mathbb{C} C^{\circ}=\mathbb{C} \bar{C} \cap\left(\mathbb{C}^{\times}\right)^{2}$ is defined by a polynomial

$$
f(z, w)=\sum a_{\left(\iota_{1}, \iota_{2}\right)} z^{\iota_{1}} w^{\iota_{2}} .
$$

Note that the condition $\mathbb{C} \bar{C} \cap \mathbb{C} E_{j}=\mathcal{P} \cap \mathbb{C} E_{j}$ implies that the Newton polygon of $f$ coincides with $\Delta$ (up to translation in $\mathbb{R}^{2}$ ). Furthermore, the intersection $\mathbb{C} \bar{C} \cap \mathbb{C} E_{j}$ is determined by $a_{\left(\iota_{1}, \iota_{2}\right)}$, with $\left(\iota_{1}, \iota_{2}\right) \in E_{j}$.

Suppose that (5.2) holds. The set $\pi_{j}^{-1}\left(\mathcal{P} \cap \mathbb{C} E_{j}\right)$ is the zero locus of a polynomial $f_{j}$ whose Newton polygon $\Delta_{f_{j}}$ is a translate of the side $E_{j}$. Multiplying $f_{j}$ by an appropriate monomial, we ensure that $\Delta_{f_{j}}=E_{j}, f_{j}=\sum a_{\left(\iota_{1}, \iota_{2}\right)}^{(j)} z^{\iota_{1}} w^{\iota_{2}}$. We have

$$
\frac{a_{v_{j-1}}^{(j)}}{a_{v_{j}}^{(j)}}=(-1)^{m_{j}} \prod_{p_{l} \in \mathbb{C} E_{j}} \varrho\left(p_{l}\right),
$$

by the Vieta theorem. Therefore, we can choose $f_{j}$ in such a way that $a_{v_{j}}^{(j)}=a_{v_{j}}^{(j+1)}$ (using the convention $a_{v_{n}}^{(n+1)}=a_{v_{n}}^{(0)}$ ) if and only if (5.2) holds.

Vice versa, if a curve with $\mathbb{C} \bar{C} \cap \partial \mathbb{C} \Delta=\mathcal{P}$ exists, then it is given by a polynomial with Newton polygon $\Delta$. Applying the Vieta theorem to the coefficients corresponding to $\partial \Delta$, we recover the condition (5.2). 
In other words, (5.2) means that the linear system defined by the divisor $\mathcal{P}$ on the (singular) elliptic curve $\partial \mathbb{C} \Delta$ is $\mathcal{O}(\Delta)$, i.e. any curve with Newton polygon $\Delta$ passing through the points $\left\{p_{j}\right\}_{j=1}^{m-1}$ also passes through $p_{m}$. By Proposition 5.3, for any subset of $m-1$ points on $\mathcal{P}$ there exists a unique remaining point with this condition. We say that $\mathcal{P}$ is a generic Menelaus configuration of $m$ points on $\partial \mathbb{C} \Delta$ if the first $m-1$ points of $\mathcal{P}$ are chosen generically on $\partial \mathbb{C} \Delta$.

Suppose now that $\mathcal{P} \subset \partial \mathbb{R} \Delta$ is a configuration of $m$ real points such that $\mathcal{P}=\operatorname{Sq}^{\Delta}(\mathcal{Q})$ for a generic Menelaus configuration $\mathcal{Q} \subset \partial \mathbb{C} \Delta$. An oriented real rational curve $\mathbb{R} \bar{C} \subset \mathbb{C} \bar{C}$ is a real curve whose normalization is isomorphic to $\mathbb{R P}^{1} \subset \mathbb{C P}^{1}$ as well as a choice of orientation on this $\mathbb{R P}^{1} \approx S^{1}$. Note that, by Jordan's theorem, such curve must be of type I. The configuration $\left(\mathrm{Sq}^{\Delta}\right)^{-1}(\mathcal{P})$ consists of real or purely imaginary points. Thus, an oriented real rational curve $\mathbb{R} \bar{C} \subset \mathbb{R} \Delta$ with Newton polygon $\Delta$ such that $\operatorname{Sq}^{\Delta}(\mathbb{C} C)$ passes through $\mathcal{P}$ has quantum index $k(\mathbb{R} C) \in \frac{1}{2} \mathbb{Z}$.

Define

$$
\sigma(\mathbb{R} C)=(-1)^{\left(m-\operatorname{Rot}_{\log }(\mathbb{R} C)\right) / 2} .
$$

Since the parities of $\operatorname{Rot}_{\log }(\mathbb{R} C)$ and $m$ coincide, we have $\sigma(\mathbb{R} C)= \pm 1$.

Remark 5.4. Note that, if $\mathbb{R} C^{\circ}$ is nodal, then its toric solitary singularities number $E(\mathbb{R} C)$ has the same parity as the number of solitary nodes of $\mathbb{R} C^{\circ}$. Thus, the Welschinger sign $w\left(\mathbb{R} C\right.$ ) (see [28]) coincides with $(-1)^{E(\mathbb{R} C)}$. Since the curve $\mathbb{R} \bar{C}$ intersects the union $\partial \mathbb{R} \Delta$ of toric divisors in $m$ distinct points and $\frac{1}{2} m \equiv \operatorname{Area}(\Delta)(\bmod 1)$ by Pick's formula, we have

$$
\sigma(\mathbb{R} C)=(-1)^{\operatorname{Area}(\Delta)-k(\mathbb{R} C)} w(\mathbb{R} C),
$$

by Theorem 3.4 .

We define

$$
R_{\Delta, k}(\mathcal{P})=\frac{1}{4} \sum_{\mathbb{R} \bar{C}} \sigma(\mathbb{R} C),
$$

where the sum is taken over all oriented real rational curves $\mathbb{R} \bar{C}$ (in particular, irreducible over $\mathbb{C}$ ) with Newton polygon $\Delta$ such that $k(\mathbb{R} \bar{C})=k$ and $\mathrm{Sq}^{\Delta}(\mathbb{C} \bar{C}) \supset \mathcal{P}$. We have the coefficient $\frac{1}{4}$ in the right-hand side of (5.5) as the group of the deck transformations of $\mathrm{Sq}^{\Delta}: \mathbb{C} \Delta \rightarrow \mathbb{C} \Delta$ is $\mathbb{Z}_{2}^{2}$, so each curve $\mathbb{R} \bar{C}$ comes in four copies with the same image $\mathrm{Sq}^{\Delta}(\mathbb{C} \bar{C})$. (Alternatively, we can take a sum over different oriented images $\mathrm{Sq}^{\Delta}(\mathbb{R} \bar{C})$ without the coefficient $\frac{1}{4}$.) Each real rational curve gives rise to two oriented real rational curves $\mathbb{R} \bar{C}$ (one for each orientation), and thus occurs in the sum in (5.5) twice.

Recall that we call a point in $\partial \mathbb{R} \Delta$ positive if it is adjacent to the quadrant $\left(\mathbb{R}_{>0}\right)^{2}$, and negative otherwise. Note that $\left(\mathrm{Sq}^{\Delta}\right)^{-1}(p)$ consists of real points if $p$ is positive and 
of purely imaginary points if $p$ is negative. Let $\lambda_{j}$ be the number of negative points in $\mathcal{P} \cap \mathbb{R} E_{j}$. Set $\lambda=\left(\lambda_{j}\right)_{j=1}^{n}$.

ThEOREM 5.5. Suppose that $\mathcal{P}=\mathrm{Sq}^{\Delta}(\mathcal{Q})$ for a generic Menelaus configuration $\mathcal{Q} \subset$ $\partial \mathbb{C} \Delta$ of $m$ points on $\partial \mathbb{C} \Delta$. If $\mathcal{P} \subset \partial \mathbb{R} \Delta$, then the number $R_{\Delta, k}(\lambda)=R_{\Delta, k}(\mathcal{P})$ is well defined. It depends only on $\Delta, k$ and $\lambda$.

If all points of $\mathcal{P}$ are negative (i.e. $m_{j}=\lambda_{j}$ ), then $R_{\Delta, k}(\lambda)$ is the number of oriented real rational curves $\mathbb{R} \bar{C}$ of quantum index $k$ contained in the positive quadrant $\mathbb{R}_{>0}^{2} \subset$ $\left(\mathbb{R}^{\times}\right)^{2}$ and passing through all points of the purely imaginary configuration $\left(\mathrm{Sq}^{\Delta}\right)^{-1}(\mathcal{P})$. For a positive point $p \in \mathcal{P}$ a curve $\mathbb{R} \bar{C}$ should pass through one of the two real points in $\left(\mathrm{Sq}^{\Delta}\right)^{-1}(p)$.

Suppose now that $\mathcal{P} \subset \partial \mathbb{R} \Delta$ itself is a generic Menelaus configuration of $m$ real points on $\partial \mathbb{C} \Delta$. (Note that, if $m$ is even, then $\mathcal{Q}$ and $\mathrm{Sq}^{\Delta}(\mathcal{Q})$ are Menelaus configurations simultaneously, but for odd $m$ the two conditions are different.) Define

$$
\widetilde{R}_{\Delta, k}(\mathcal{P})=\sum_{\mathbb{R} \bar{C}} \sigma(\mathbb{R} C)
$$

where the sum is taken over all oriented real rational curves $\mathbb{R} \bar{C}$ of quantum index $k$ with Newton polygon $\Delta$ and $k(\mathbb{R} C)=k$ passing through $\mathcal{P}$. Let

$$
\Delta_{d}=\operatorname{Conv}\{(0,0),(d, 0),(0, d)\} .
$$

We have $\mathbb{C} \Delta_{d}=\mathbb{C P}^{2}$.

Example 5.6. The curves $\mathbb{R} C$ with Newton polygon $\Delta_{2}$ are projective conics. In this case $n=3, m_{1}=m_{2}=m_{3}=2$ and $m=6$, and for any generic Menelaus configuration $\mathcal{P} \subset \partial \mathbb{R P}^{2}$ we have a unique conic through $\mathcal{P}$. This gives us two oriented curves through $\mathcal{P}$ of opposite quantum index.

We may assume (applying the reflections in the $x$ - and $y$-axes if needed) that $\mathcal{P}$ contains a positive point in the $x$-axis and a positive point in the $y$-axis. As the positivity of the last point of $\mathcal{P}$ will be determined by the Menelaus condition, we have three possibilities for the non-decreasing sequence $\lambda=\left(\lambda_{1}, \lambda_{2}, \lambda_{3}\right)$. The possible values of $k(\mathbb{R} C)$ are listed Table 5.1; cf. Figure 3.2.

In particular, in this case $\widetilde{R}_{\Delta_{2}, k}(\mathcal{P})$ changes for different configurations with the same $\lambda$ for the two last rows of Table 5.1. Thus, the numbers $\widetilde{R}_{\Delta, k}(\mathcal{P})$ may vary when we deform $\mathcal{P}$.

Define

$$
\widetilde{R}_{\Delta, \text { even }}(\mathcal{P})=\sum_{k \in m / 2+2 \mathbb{Z}} \widetilde{R}_{\Delta, k}(\mathcal{P}) \quad \text { and } \quad \widetilde{R}_{\Delta, \text { odd }}(\mathcal{P})=\sum_{k \in m / 2+1+2 \mathbb{Z}} \widetilde{R}_{\Delta, k}(\mathcal{P}) \text {. }
$$




\begin{tabular}{||c|c|c||c||}
\hline$\lambda_{1}$ & $\lambda_{2}$ & $\lambda_{3}$ & $k(\mathbb{R} C)$ \\
\hline 0 & 0 & 0 & \pm 2 \\
\hline 0 & 0 & 2 & \pm 1 or 0 \\
\hline 0 & 1 & 1 & \pm 1 or 1 \\
\hline
\end{tabular}

Table 5.1. Quantum indices for conics.

Theorem 5.7. The numbers $\widetilde{R}_{\Delta_{d} \text {,even }}(\mathcal{P})$ and $\widetilde{R}_{\Delta_{d} \text {,odd }}(\mathcal{P})$ do not depend of $\mathcal{P}$ as long as $d$ is even and all the points of $\mathcal{P}$ are positive (i.e. $\lambda=(0,0,0))$.

\subsection{Refined real and refined tropical enumerative geometry}

We return to the study of the invariant $R_{\Delta, k}$ from Theorem 5.5.

Definition 5.8. The sum

$$
R_{\Delta}(\lambda)=\sum_{k=-\operatorname{Area}(\Delta)}^{\operatorname{Area}(\Delta)} R_{\Delta, k}(\lambda) q^{k}
$$

is called the real refined enumerative invariant of $\left(\mathbb{R}^{\times}\right)^{2}$ in degree $\Delta$.

If $\lambda=0$ (i.e. all $\lambda_{j}=0$ ), then all points of $\left(\mathrm{Sq}^{\Delta}\right)^{-1}(\mathcal{P})$ are real. In such case, we use the notation $R_{\Delta, k}=R_{\Delta, k}(0)$ and $R_{\Delta}=R_{\Delta}(0)$.

Recall that the Block-Göttsche invariant [1] is a symmetric (with respect to the substitution $q \mapsto q^{-1}$ ) Laurent polynomial with positive integer coefficients. This polynomial is responsible for the enumeration of the tropical curves with the Newton polygon $\Delta$ of genus $g$, passing through a generic collection of points in $\mathbb{R}^{2}$; see [11]. The expression $N_{\Delta}^{\partial, \text { trop }}$ defined by (6.22) may be viewed as the counterpart of $N_{\text {trop }}^{\Delta, 0}$ defined in [1]. In this counterpart, the tropical curves pass through a collection of $m$ points on the boundary of the toric tropical surface $\mathbb{T} \Delta$ which are generic among those satisfying the tropical Menelaus condition (6.17).

THEOREM 5.9.

$$
R_{\Delta}=\left(q^{1 / 2}-q^{-1 / 2}\right)^{m-2} N_{\Delta}^{\partial, \text { trop }} .
$$

Corollary 5.10. The number $N_{\Delta}^{\partial, \mathbb{C}}$ of complex rational curves in $\mathbb{C} \Delta$ with Newton polygon $\Delta$ passing through $\mathcal{P}$ is determined by $R_{\Delta}$.

Proof. By [18], the number $N_{\Delta}^{\partial, \mathbb{C}}$ coincides with the value of $N_{\Delta}^{\partial \text {,trop }}$ at $q=1$.

Let us reiterate that $R_{\Delta}$ accounts only for curves in $\left(\mathbb{C}^{\times}\right)^{2}$ defined over $\mathbb{R}$. 


\subsection{Holomorphic disk interpretation}

Recall that an orientation of a real rational curve $\mathbb{R} C$ defines a connected component $S \subset \mathbb{C} \widetilde{C} \backslash \mathbb{R} \widetilde{C}$. Let $D$ be the topological closure of the image of $S$ in $\mathbb{C} \Delta$. The disk $D \subset \mathbb{C} \Delta$ is a holomorphic disk whose boundary $\partial D=\mathbb{R} C$ is contained in the Lagrangian subvariety $\mathbb{R} \Delta \subset \mathbb{C} \Delta$.

Let $L$ be the topological closure of the quadrant $\mathbb{R}_{>0}^{2}$ in $\mathbb{C} \Delta$. Note that $L$ is a Lagrangian subvariety of $\mathbb{C} \Delta$ with boundary. The image $\mathrm{Sq}^{\Delta}(D)$ is a holomorphic disk whose boundary is contained in $L$.

Thus, the expression (5.9) may also be interpreted as a refined enumeration of holomorphic disks with boundary in $L$, passing through $\mathcal{P}$, and tangent to $\partial \mathbb{R} \Delta$ at the points of $\mathcal{P}$.

These disks are images under $\mathrm{Sq}^{\Delta}$ of disks $D$ with boundary in $\mathbb{R} \Delta$ and

$$
\mathrm{Sq}^{\Delta}(D) \cap \partial \mathbb{C} \Delta=\mathcal{P} \subset \partial \mathbb{R} \Delta .
$$

Let $\widehat{\mathbb{C} \Delta}$ be the result of blowing-up the toric variety $\mathbb{C} \Delta$ at $\mathcal{P}$. Let $\hat{L}=\overline{\left(\mathbb{R}^{\times}\right)^{2}} \backslash \widehat{\partial \mathbb{R} \Delta}$, where $\overline{\left(\mathbb{R}^{\times}\right)^{2}}$ is the topological closure of $\left(\mathbb{R}^{\times}\right)^{2}$ in $\widehat{\mathbb{C} \Delta}$ and $\widehat{\partial \mathbb{R} \Delta}$ is the proper transform of $\partial \mathbb{R} \Delta$ in $\widehat{\mathbb{C} \Delta}$. Then, a holomorphic disk $D$ lifts to a holomorphic disk $\widehat{D}$ with boundary in the non-compact Lagrangian subvariety $\hat{L} \subset \widehat{\mathbb{C} \Delta}$ without boundary. Furthermore, the Maslov index of $\widehat{D}$ is zero.

\section{Proofs}

\subsection{Proof of Proposition 3.3 and Theorems 3.1, 3.4 and 4.7}

Consider the map Arg: $\left(\mathbb{C}^{\times}\right)^{2} \rightarrow(\mathbb{R} / 2 \pi \mathbb{Z})^{2}$ defined by

$$
\operatorname{Arg}(z, w)=(\arg (z), \arg (w)),
$$

and the map 2Arg: $\left(\mathbb{C}^{\times}\right)^{2} \rightarrow(\mathbb{R} / \pi \mathbb{Z})^{2}$ obtained by multiplication of Arg by 2 , in other words a composition of Arg with with the quotient map $(\mathbb{R} / 2 \pi \mathbb{Z})^{2} \rightarrow(\mathbb{R} / \pi \mathbb{Z})^{2}$. The involution of complex conjugation in $\left(\mathbb{C}^{\times}\right)^{2}$ descends to $(\mathbb{R} / \pi \mathbb{Z})^{2}$ as the involution

$$
\sigma:(a, b) \longmapsto(-a,-b) .
$$

Denote by

$$
P=(\mathbb{R} / \pi \mathbb{Z})^{2} / \sigma
$$

the quotient space. The orbifold $P$ is the so-called pillowcase. The projections of the four points $(0,0),\left(\frac{1}{2} \pi, 0\right),\left(0, \frac{1}{2} \pi\right)$ and $\left(\frac{1}{2} \pi, \frac{1}{2} \pi\right)$ form the $\mathbb{Z}_{2}$-orbifold locus of $P$ (the corners 
of the pillowcase). All other points are smooth. We denote by $0 \in P$ the origin of $P$, i.e. the projection of $(0,0)$. Note that $(2 \mathrm{Arg})^{-1}(0,0)=\left(\mathbb{R}^{\times}\right)^{2}$. The product volume form on $(\mathbb{R} / \pi \mathbb{Z})^{2}$ defines the volume form $d \operatorname{Vol}_{P}$ on the smooth points of the orbifold $P$, since the involution $\sigma$ is orientation preserving.

Let $\mathbb{R} C$ be a real curve of type I with real or purely imaginary coordinate intersection. Consider the surface $S^{\circ}=S \backslash \nu^{-1}(\partial \mathbb{C} \Delta)$, where $S$ is the component of $\mathbb{C} \widetilde{C} \backslash \mathbb{R} \widetilde{C}$ corresponding to the orientation of $\mathbb{R} C$, and $\nu$ is the normalization map (2.1). Denote by

$$
\beta: S^{\circ} \longrightarrow P
$$

the composition of the map $\left.2 \operatorname{Arg}\right|_{S^{\circ}}: S^{\circ} \rightarrow(\mathbb{R} / \pi \mathbb{Z})^{2}$ and the projection $(\mathbb{R} / \pi \mathbb{Z})^{2} \rightarrow P$.

Let $p \in P$ be a regular point of $\beta$. A point $q \in \beta^{-1}(p)$ is called positive (resp. negative) if locally near $q$ the map $\beta$ is an orientation-preserving (resp. orientation-reversing) open embedding. The difference between the number of positive and negative points in $\beta^{-1}(p)$ is called the degree at $p$. A priori, since $\beta$ is a non-proper map, the degree at different points could be different.

Lemma 6.1. We have

$$
\operatorname{Area}_{\log }(\mathbb{R} C)=\int_{S^{\circ}} \beta^{*} d \operatorname{Vol}_{P} .
$$

Furthermore, the degree of $\beta$ at a generic point of $P$ is $2 k(\mathbb{R} C)$.

Proof. Consider the form

$$
\begin{aligned}
\frac{d x}{x} \wedge \frac{d y}{y}= & (d \log |x|+i d \arg (x)) \wedge(d \log |y|+i d \arg (y)) \\
= & d \log |x| \wedge d \log |y|-d \arg (x) \wedge d \arg (y) \\
& +i(d \log |x| \wedge d \arg (y)+d \arg (x) \wedge d \log |y|)
\end{aligned}
$$

on $\left(\mathbb{C}^{\times}\right)^{2}$. As it is a holomorphic 2 -form, it must restrict to the zero form on any holomorphic curve in $\left(\mathbb{C}^{\times}\right)^{2}$. In particular, the real part of this form must vanish everywhere on $S^{\circ}$, so that $d \log |x| \wedge d \log |y|=d \arg (x) \wedge d \arg (y)$ on $S^{\circ}$, and thus (6.4) holds; cf. [20].

The smooth map $\beta: S^{\circ} \rightarrow P$ extends to a continuous map

$$
\bar{\beta}: \bar{S} \longrightarrow P
$$

for a surface with boundary $\bar{S} \supset S^{\circ}$ such that $S^{\circ}=\bar{S} \backslash \partial \bar{S}$. Each $p \in \mathbb{C} \bar{C} \cap \mathbb{C} E_{j}$ corresponds to a geodesic in $(\mathbb{R} / \pi \mathbb{Z})^{2}$ in the direction parallel to $\vec{n}\left(E_{j}\right)$ for a side $E_{j} \subset \partial \Delta$; cf. [21]. Since $\mathrm{Sq}^{\Delta}(p) \in \mathbb{R} \Delta$, the corresponding geodesic passes through two of the points $(0,0)$, 
$\left(\frac{1}{2} \pi, 0\right),\left(0, \frac{1}{2} \pi\right)$ and $\left(\frac{1}{2} \pi, \frac{1}{2} \pi\right)$. The image of this circle in $P$ is a geodesic segment connecting the corresponding $\mathbb{Z}_{2}$-orbifold points of $P$.

Thus, $\bar{\beta}(\bar{S})$ is a 2 -cycle in $P$ and it covers a generic point $l$ times (counted algebraically), where $l$ is a number independent of the choice of the generic point. But then

$$
\int_{S^{\circ}} \beta^{*} d \operatorname{Vol}_{P}=l \operatorname{Area}(P)=\frac{l \pi^{2}}{2}
$$

Since we have already proved (6.4), we get

$$
l=2 \frac{\operatorname{Area}(\mathbb{R} C)}{\pi^{2}}=2 k(\mathbb{R} C)
$$

(the last equality is the definition of $k(\mathbb{R} C)$ ).

Note that this lemma implies Proposition 3.3.

Proof of Theorem 3.1. We have $k(\mathbb{R} C) \in \frac{1}{2} \mathbb{Z}$, since $2 k(\mathbb{R} C)$ is the degree of $\beta$ at a generic point of $P$ by Lemma 6.1 . Let $\tilde{a} \in(\mathbb{R} / \pi \mathbb{Z})^{2}$ be a generic point and $a \in P$ be the point corresponding to $\tilde{a}$. The inverse image $\beta^{-1}(a)$ consists of points of $S^{\circ}$ mapped to $\tilde{a}$ or $\sigma(\tilde{a})$. If $2 \operatorname{Arg}(p)=-\tilde{a}$ for $p \in S^{\circ}$, then $2 \operatorname{Arg}(\operatorname{conj}(p))=\tilde{a}$, where $\operatorname{conj}(p) \in \operatorname{conj}\left(S^{\circ}\right)$. Thus we have a one-to-one correspondence between the sets $\beta^{-1}(a)$ and $R=(2 \operatorname{Arg})^{-1}(\tilde{a}) \cap \mathbb{C} C^{\circ}$.

Consider the continuous involution $\operatorname{conj}_{\tilde{a}}: \mathbb{C} \Delta \rightarrow \mathbb{C} \Delta$ which extends the involution of $\left(\mathbb{C}^{\times}\right)^{2}$ defined by $z \mapsto e^{i \tilde{a}} \operatorname{conj}(z)$. Note that the fixed point locus of this involution in $\left(\mathbb{C}^{\times}\right)^{2}$ coincides with $(2 \operatorname{Arg})^{-1}(\tilde{a}) ;$ cf. [20]. Note that

$$
R \subset \mathbb{C} C^{\circ} \cap \operatorname{conj}_{\tilde{a}}\left(\mathbb{C} C^{\circ}\right),
$$

while $R \backslash\left(\mathbb{C} C^{\circ} \cap \operatorname{conj}_{a}\left(\mathbb{C} C^{\circ}\right)\right)$ consists of pairs of points interchanged by the involution $\operatorname{conj}_{\tilde{a}}$. For generic $\tilde{a}$, the curve $\operatorname{conj}_{\tilde{a}}\left(\mathbb{C} C^{\circ}\right)$ is transverse to $\mathbb{C} C^{\circ}$, while

$$
\operatorname{conj}_{\tilde{a}}\left(\mathbb{C} C^{\circ}\right) \cap \mathbb{C} C^{\circ} \cap \partial \mathbb{C} \Delta=\varnothing .
$$

Thus, the number of points in $R$ is not greater than $\#\left(\mathbb{C} C^{\circ} \cap \operatorname{conj} j_{\tilde{a}}\left(\mathbb{C} C^{\circ}\right)\right)$, while we have $\#\left(\mathbb{C} C^{\circ} \cap \operatorname{conj}_{\tilde{a}}\left(\mathbb{C} C^{\circ}\right)\right)=2 \operatorname{Area}(\Delta)$ by the Kushnirenko-Bernstein theorem [16]. Thus, the degree of $\beta$ takes values between $-2 \operatorname{Area}(\Delta)$ and $2 \operatorname{Area}(\Delta)$. Also, we have $\# R=2 \operatorname{Area}(\Delta)$.

Proof of Theorem 3.4. Let us compute the degree of the map (6.6) at a generic point $a \in P$ close to the origin $0 \in P$. The set $\bar{\beta}^{-1}(0) \cap S^{\circ}$ contributes $2 E$ to the degree of $\bar{\beta}$, as the intersection number gets doubled when we pass from $(\mathbb{R} / \pi \mathbb{Z})^{2}$ to $P$.

Note that the set $S_{\mathbb{R}}=\bar{\beta}^{-1}(0) \cap \partial \bar{S}$ can be thought of as the topological closure of $\mathbb{R} C^{\circ}$ in $\bar{S}$, by our assumption of transversality to $\partial \mathbb{R} \Delta$. Consider a non-vanishing tangent 
vector field $\gamma$ on $\mathbb{R} \widetilde{C}^{\circ}$ such that it extends to a tangent vector field on $\mathbb{R} \widetilde{C}$ with simple zeros at $\mathbb{R} \widetilde{C} \backslash \mathbb{R} \widetilde{C}^{\circ}$. Our condition on the zeros of $\gamma$ implies that $\pm i \gamma$ is consistent with a trivialized regular neighborhood $U \approx S_{\mathbb{R}} \times\left[0,1\right.$ ) (we take $i \gamma$ on the components of $S_{\mathbb{R}}$ where $\gamma$ agrees with the complex orientation of $\mathbb{R} C^{\circ}$, and $-i \gamma$ otherwise). The lift $\tilde{\beta}_{\varepsilon}$ of $\left.\bar{\beta}\right|_{S_{\mathbb{R}} \times\{\varepsilon\}}$ to $\mathbb{R}^{2} /\{(x, y) \sim(-x,-y)\}$ is approximated (to the first order by $\varepsilon$ ) by $\varepsilon \gamma$ for small $\varepsilon>0$. Thus, the linking number of the image of $\tilde{\beta}_{\varepsilon}$ and $(0,0) \in \mathbb{R}^{2} /\{(x, y) \sim(-x,-y)\}$ is $\operatorname{Rot}_{\log }(\mathbb{R} C)$. Thus, $S_{\mathbb{R}}$ contributes $-\operatorname{Rot}_{\log }(\mathbb{R} C)$ to the degree of $\bar{\beta}$. We have the appearance of the negative sign, since the basis made of the vectors $v_{1}, v_{2}, i v_{1}$ and $i v_{2}$ is negatively-oriented in $\mathbb{C}^{2}$ whenever the vectors $v_{1}$ and $v_{2}$ are linearly independent over $\mathbb{C}$. Thus, a positive rotation in $(i \mathbb{R})^{2}$ (and therefore also in $P$ ) corresponds to a negative contribution to the degree of $\bar{\beta}$.

Proof of Theorem 4.7. The map (4.3) gives the lift of $\left.\beta\right|_{S^{\circ}}$ to the universal covering $\mathbb{C}^{2}$ of $\left(\mathbb{C}^{\times}\right)^{2}$, after rescaling each coordinate by $\pi$. Thus, the signed area of $\beta\left(S^{\circ}\right)$ coincides with $\pi^{2}$ Area $(\Sigma(\mathbb{R} C))$. Lemma 6.1 now implies that $k(\mathbb{R} C)=$ Area $\Sigma(\mathbb{R} C)$. For $(a, b) \in \mathbb{Z}^{2}$ and $\varepsilon \in \widetilde{\mathbb{R P}}^{1} \backslash \widetilde{\mathbb{Q P}}^{1}$ we consider a point $p_{\varepsilon}$ obtained by a small translation of $(a, b)$ in the direction of $\varepsilon$. A point of $S^{\circ}$ mapped to $p_{\varepsilon}$ by the lift of $\beta$ must correspond to a point of $\mathbb{R} C^{\circ}$ of real index $(a, b)$, which is either singular or has $\varepsilon$ as image of its logarithmic Gauss map. Summing up the contributions of all such points, we get (4.10).

\subsection{Spaces $\mathcal{M}_{\Delta}$ and $\mathcal{M}_{\partial \Delta}$.}

To prove Theorem 5.5, we consider parameterized curves in $\mathbb{C} \Delta$, i.e. maps from abstract curves to $\mathbb{C} \Delta$. For our purposes, it is convenient to consider not only irreducible rational curves as sources for these maps, but also the so-called stable rational curves with marked points (cf. [6] and [14]). These are (perhaps reducible) nodal curves made of rational irreducible components, so that the dual graph (formed by irreducible components as vertices and nodes as edges) is a tree. The marked points are required to be distinct and disjoint from the nodes. If an irreducible component of such curve is adjacent to $j$ other components and contains less than $3-j$ marked points, then it can be contracted to a point (a smooth point if $j=1$ and a node if $j=2$ ) in a new nodal curve, so that the images of the marked points are still distinct and disjoint from the nodes. These operations generate an equivalence relation on the space of stable rational curves with marked points.

We may consider compact stable rational curves (formed by compact rational irreducible components with marked points), as well as punctured stable rational curves obtained by puncturing a compact stable rational curve at all its marked points. Clearly, any compact stable rational curve corresponds to a punctured stable rational curve, and 
vice versa. It is convenient for us not to order marked points, i.e. marked points are treated as an $m$-element subset of a stable rational curve.

By stable rational curves in $\mathbb{C} \Delta$, we mean continuous maps from compact stable rational curves with $m$ marked points to $\mathbb{C} \Delta$ such that the restriction to each irreducible component is holomorphic, and the marked points are mapped to $\partial \mathbb{C} \Delta$. Two such maps are considered to be equivalent if their source curves are equivalent, and the equivalence agrees with the map, i.e. only a component where our map is constant can be contracted. Clearly, the images of equivalent stable curves in $\mathbb{C} \Delta$ coincide. Any stable rational curve in $\mathbb{C} \Delta$ corresponds to a proper map from a punctured stable rational curve (with $m$ punctures) to $\left(\mathbb{C}^{\times}\right)^{2}$. We say that a stable rational curve in $\mathbb{C} \Delta$ is real if its source is equipped with an antiholomorphic involution agreeing with the complex conjugation in $\mathbb{C} \Delta$.

Let $\mathcal{M}_{\Delta}$ be the space of real stable rational curves $h: \mathbb{C} \widetilde{C} \rightarrow \mathbb{C} \Delta$ such that the image $h(\mathbb{C} \widetilde{C})$ is transversal to the boundary $\partial \mathbb{C} \Delta$, i.e. $h(\mathbb{C} \widetilde{C}) \cap \partial \mathbb{C} \Delta$ consists of $m$ distinct points disjoint from the pairwise intersection points of toric divisors of $\partial \mathbb{C} \Delta$. Let $\mathcal{M}_{\partial \Delta}$ be the space of conj-invariant configurations $\mathcal{P}$ of $m$ distinct points in $\partial \mathbb{C} \Delta$ with $m_{j}$ points in $\mathbb{C} E_{j}, m=\sum_{j} m_{j}=\#\left(\partial \Delta \cap \mathbb{Z}^{2}\right)$, such that there exists a Menelaus configuration $\mathcal{Q}$ of $m$ points in $\partial \mathbb{C} \Delta$ with $\mathcal{P}=\operatorname{Sq}(\mathcal{Q})$. This space is an open set in the real part of the product of $m-1$ copies of $\mathbb{C}^{\times}$(for a choice of a real structure on that product). Thus, $\mathcal{M}_{\partial \Delta}$ is a smooth manifold of dimension $m-1$.

Proposition 6.2. The map

$$
\mathrm{ev}: \mathcal{M}_{\Delta} \longrightarrow \mathcal{M}_{\partial \Delta}
$$

sending a curve $h: \mathbb{C} \widetilde{C} \rightarrow \mathbb{C} \Delta$ to the configuration $\mathrm{Sq}^{\Delta}(h(\mathbb{C} \widetilde{C})) \cap \partial \mathbb{C} \Delta$ is a proper smooth map between $(m-1)$-dimensional smooth real manifolds.

Proof. First, we note that $\mathcal{M}_{\Delta}$ is a smooth manifold. This follows from smoothness of the space $\overline{\mathcal{M}}_{0, m}$ of compact stable rational curves with $m$ marked points (see [6]). Indeed, for $h \in \mathcal{M}_{\Delta}$ we have a local diffeomorphism between deformations of the source of $h$ in the space of compact stable rational curve and a neighborhood of the corresponding point in $\overline{\mathcal{M}}_{0, m}$, once we order all marked points of the source of $h$ arbitrarily. Since $h$ is determined by the position of zeros and poles (marked points) on its source, up to a coordinatewise multiplication in $\left(\mathbb{C}^{\times}\right)^{2}$, locally $\mathcal{M}_{\Delta}$ is a $\left(\mathbb{R}^{\times}\right)^{2}$-fibration over $\overline{\mathcal{M}}_{0, m}$, and thus smooth. Furthermore, $\operatorname{dim} \mathcal{M}_{\Delta}=2+\operatorname{dim} \overline{\mathcal{M}}_{0, m}=m-1$.

Recall that stable rational curves with ordered marked points form a compact space (see [6] and [14]). Suppose that we have a sequence of curves in $\mathcal{M}_{\Delta}$ such that their image is converging to a point in $\mathcal{M}_{\partial \Delta}$. By compactness, there exists a stable rational curve 
$h: A \rightarrow \mathbb{C} \Delta$ which is an accumulation point for this sequence. Note that $h$ is real, since $A$ acquires an antiholomorphic involution from the approximating real stable rational curves. If no components of $A$ are mapped to $\partial \mathbb{C} \Delta=\mathbb{C} \Delta \backslash\left(\mathbb{C}^{\times}\right)^{2}$, then $h(A) \cap\left(\mathbb{C}^{\times}\right)^{2}$ has $\Delta$ as its Newton polygon, otherwise the Newton polygon is a subpolygon of $\Delta$. In the first case, $h \in \mathcal{M}_{\Delta}$.

Thus, to establish the properness of the map ev, it is enough to show that no irreducible component $K \subset A$ can be mapped to $\partial \mathbb{C} \Delta$. Note that $h(K)$ must be contained in a single boundary divisor of $\mathbb{C} \Delta$, and intersects two other boundary divisors at two points $v_{1}$ and $v_{2}$, corresponding to two vertices of $\Delta$. In particular the points $v_{j}, j=1,2$, are disjoint from any configuration of $\mathcal{M}_{\partial \Delta}$. Thus, each point in the inverse image $h^{-1}\left(v_{j}\right)$ must be a node of $A$, otherwise any perturbation of $h$ in the class of stable rational curves would intersect $\partial \mathbb{C} \Delta$ at a point close to $v_{j}$. But the graph $\Gamma$ dual to the arrangement of components of $A$ is a tree. Thus, there must exist a component $K$ adjacent to a single node. If this component is mapped to $\partial \mathbb{C} \Delta$, we get a contradiction. If not, we form a new tree $\Gamma^{\prime}$ by removing the 1-valent vertex of $\Gamma$ corresponding to $K$ and proceed inductively. Eventually, we find a component of $A$ such that at most one of its adjacent components is mapped to $\partial \mathbb{C} \Delta$. Thus, at most one of the vertex points $v_{1}$ and $v_{2}$ corresponding to this component can have the preimage consisting entirely of nodes, and we get a contradiction.

Proposition 6.3. For a generic configuration $\mathcal{P} \subset \mathcal{M}_{\partial \Delta}$ the set $\mathrm{ev}^{-1}(\mathcal{P})$ is finite and consists of immersions of irreducible rational curves to $\left(\mathbb{C}^{\times}\right)^{2}$.

For a generic path $\left\{\mathcal{P}_{t}\right\}_{t \in[0,1]} \subset \mathcal{M}_{\partial \Delta}$ connecting two generic configurations $\mathcal{P}_{0}$ and $\mathcal{P}_{1}$ the inverse image $\mathrm{ev}^{-1}\left(\left\{\mathcal{P}_{t}\right\}_{t \in[0,1]}\right)$ consists of curves with at most two irreducible components. Furthermore, in the case of non-irreducible curves, both components are immersed real rational curves intersecting transversely with each other.

Proof. Each component of a non-irreducible stable rational curve in $\mathbb{C} \Delta$ is itself a stable rational curve. Thus, by the dimension computation of Proposition 6.2, the dimension of the locus of $\mathcal{M}_{\Delta}$ corresponding to curves with $a$ components is $m-a$. Note that to deform a single marked point from $p$ to $p^{\prime}$ we can multiply coordinate functions by rational functions with zeros and poles only at $p$ and $p^{\prime}$. A non-immersed point of $\mathbb{C} C^{\circ}$ corresponds to a common zero of the differentials of the coordinate functions. Clearly, the coordinate functions can be deformed independently of each other. Thus, a generic rational curve in $\left(\mathbb{C}^{\times}\right)^{2}$ with Newton polygon $\Delta$ is immersed.

The set of curves with two components such that one of them is not smoothly immersed or such that the components intersect non-transversely has codimension 1 in the set of reducible curves, and thus it has codimension 2 in $\mathcal{M}_{\Delta}$. 


\subsection{Invariance of $R_{\Delta, k}(\mathcal{P})$ under deformations of $\mathcal{P}$ disjoint from the image of reducible curves}

Consider the space $\mathcal{M}_{\partial \Delta, \lambda} \subset \mathcal{M}_{\partial \Delta}$ of real configurations $\mathcal{P} \subset \mathbb{R} \Delta$ with $\lambda_{j}$ points in

$$
\mathbb{R} E_{j} \backslash \overline{\left(\mathbb{R}_{>} 0\right)^{2}} .
$$

Note that the space $\mathcal{M}_{\partial \Delta, \lambda}$ is connected. Any curve in $\operatorname{ev}^{-1}\left(\mathcal{M}_{\partial \Delta, \lambda}\right)$ satisfies the hypothesis of Theorem 3.1, thus its quantum index is well defined (once the orientation of each irreducible component is fixed). Let $\mathcal{P}, \mathcal{P}^{\prime} \in \mathcal{M}_{\partial \Delta, \lambda}$ and $\gamma=\left\{\mathcal{P}_{t}\right\}_{t \in[0,1]} \in \mathcal{M}_{\partial \Delta, \lambda}$ be a smooth generic path connecting two generic configurations $\mathcal{P}=\mathcal{P}_{0}$ and $\mathcal{P}^{\prime}=\mathcal{P}_{1}$ from $\mathcal{M}_{\partial \Delta, \lambda}$.

Let $\mathcal{C} \subset \mathrm{ev}^{-1}(\gamma)$ be a connected component. Recall that ev is a proper smooth map between manifolds of the same dimension, by Proposition 6.2. By Sard's theorem, since $\left\{\mathcal{P}_{t}\right\}$ is a generic smooth path, the component $\mathcal{C}$ is a smooth 1-manifold. Thus, it is diffeomorphic to a compact interval or to a circle. By Proposition 6.3, all but finitely many values of $t$ correspond to the configurations $\mathcal{P}_{t}$ such that $\mathrm{ev}^{-1}\left(\mathcal{P}_{t}\right)$ consists of smoothly immersed irreducible curves. In this case, we may identify a stable rational curve with its image in $\mathbb{C} \Delta$. Define $\mathcal{R}_{\Delta, k}\left(\mathcal{P}_{t}\right)$ as the subset of $\mathrm{ev}^{-1}\left(\mathcal{P}_{t}\right)$ consisting of curves with quantum index $k$.

Proposition 6.4. If $\mathcal{C}$ is disjoint from the locus of reducible curves in $\mathcal{M}_{\Delta}$, then

$$
\sum_{\mathbb{R} \bar{C} \in \mathcal{R}_{\Delta, k}\left(\mathcal{P}_{0}\right) \cap \mathcal{C}} \sigma(\mathbb{R} C)=\sum_{\mathbb{R} \bar{C} \in \mathcal{R}_{\Delta, k}\left(\mathcal{P}_{1}\right) \cap \mathcal{C}} \sigma(\mathbb{R} C) .
$$

Note that our definitions of the sign $\sigma(\mathbb{R} C)$ depends on the orientation of $\mathbb{R} C$ (recall that $\mathcal{M}_{\Delta}$ is defined as the space of oriented real curves). With the help of (5.4), we may reformulate the proposition in terms of Welschinger's signs $w(\mathbb{R} C)=(-1)^{E(\mathbb{R} C)}$ which are independent of the choice of the orientation of $\mathbb{R} C$ as

$$
\sum_{\mathbb{R} \bar{C} \in \mathcal{R}_{\Delta, k}\left(\mathcal{P}_{0}\right) \cap \mathcal{C}} w(\mathbb{R} C)=\sum_{\mathbb{R} \bar{C} \in \mathcal{R}_{\Delta, k}\left(\mathcal{P}_{1}\right) \cap \mathcal{C}} w(\mathbb{R} C),
$$

since the quantum index $k$ is constant on $\mathcal{C}$. In this form, Proposition 6.4 may be viewed as a version of Welschinger's invariance. To prove the proposition, we need to recall orientation constructions for the space of sections of real vector bundles over an oriented real rational curve. We do it in a series of auxiliary lemmas.

Let $\pi_{A}$ be a line bundle of degree $a \geqslant 0$ over an oriented real rational (non-empty) curve $\mathbb{R} K, \xi$ be its section with distinct real zeros $x_{1}, \ldots, x_{a} \in \mathbb{R} K$, and $x_{0} \in \mathbb{R} K$ be a point such that $x_{0}, x_{1}, \ldots, x_{a}, \in \mathbb{R} K$ are numbered in the order consistent with the orientation 
of $\mathbb{R} K$. For each $j=1, \ldots, a$, consider a section $\xi_{j}$ of $\pi_{A}$ whose zeros are obtained from $\left\{x_{l}\right\}_{l=1}^{a}$ by moving the point $x_{j}$ in the positive direction in $\mathbb{R} K$, while leaving the other points unchanged. In addition, we assume that $\xi\left(x_{0}\right)$ and $\xi_{j}\left(x_{0}\right)$ are positive multiples of each other.

Lemma 6.5. For a real algebraic line bundle $\pi_{A}$ of degree $a \geqslant 0$ over an oriented rational real curve $\mathbb{R} K$ the sections $\xi, \xi_{1}, \ldots, \xi_{a}$ form a basis of $\Gamma\left(\pi_{A}\right)$. The orientation or $A$ of the vector space $\Gamma\left(\pi_{A}\right)$ determined by $\xi, \xi_{1}, \ldots, \xi_{a}$ depends only on the orientation of the curve $\mathbb{R} K$, if a is odd. If a is even, then or $_{A}$ depends on the orientation of the curve $\mathbb{R} K$ as well as the orientation of $\pi_{A}$ determined by the non-zero vector $\xi\left(x_{0}\right)$.

Proof. Since the points $x_{1}, \ldots, x_{a}$ are distinct, any linear combination of the sections $\xi_{j}$ vanishing at $x_{l}$ must have zero as coefficient of $\xi_{j}$. The sections $\xi_{j}$ along with $\xi$ form a basis, since $\operatorname{dim} \Gamma\left(\pi_{A}\right)=a+1$ and $\xi \neq 0$. All configurations of $a+1$ distinct points in $\mathbb{R} K$ in the same cyclic order are isotopic to each other. Reversing the orientation of $\pi_{A}$ over $x_{0}$ results in reversing the sign for all sections of our basis. The resulting basis is of the same sign if and only if $a+1$ is even.

Let now $\pi_{E}$ be a real algebraic 2-dimensional vector bundle of degree $d$ over $\mathbb{R} K$. We assume that $\pi_{E}$ is generated by global sections. Since $\pi_{E}$ decomposes into the sum of two line bundles (as $\mathbb{R} K$ is rational), this assumption is equivalent to the non-negativity of the degrees of both line bundles. In particular, we have $d \geqslant 0$ and $\operatorname{dim} \Gamma\left(\pi_{E}\right)=d+2 \geqslant 2$.

Suppose that $\pi_{A}: \mathbb{R} A \rightarrow \mathbb{R} K$ is a (1-dimensional) sub-bundle of $\pi_{E}$ of non-negative degree $a$. Let $\pi_{E / A}$ be the corresponding quotient line bundle. We get the following short exact sequence

$$
0 \longrightarrow \Gamma\left(\pi_{A}\right) \longrightarrow \Gamma\left(\pi_{E}\right) \longrightarrow \Gamma\left(\pi_{E / A}\right) \longrightarrow 0
$$

which allows us to combine orientations of $\Gamma\left(\pi_{A}\right)$ and $\Gamma\left(\pi_{E / A}\right)$ into an orientation of $\Gamma\left(\pi_{E}\right)$. Note that, if $a$ is even, then changing the sign of $\xi\left(x_{0}\right)$ also changes or $A$. Thus, the following statement is thus a corollary of Lemma 6.5.

COROllary 6.6. Let $\pi_{E}$ be a real algebraic 2-dimensional vector bundle of degree $d$ generated by global sections. Let $\pi_{A}$ be its real algebraic 1-dimensional sub-bundle of degree $a \geqslant 0$.

If $d$ is even, then the orientation of $\Gamma\left(\pi_{E}\right)$ is defined by $\pi_{A}$ together with an orientation of the bundle $\pi_{E}$.

If $d$ and a are odd, then the orientation of $\Gamma\left(\pi_{E}\right)$ is defined by $\pi_{A}$ together with an orientation of the bundle $\pi_{E / A}$.

If $d$ is odd while a is even, then the orientation of $\Gamma\left(\pi_{E}\right)$ is defined by $\pi_{A}$ together with an orientation of the bundle $\pi_{A}$. 
Denote the resulting orientation of $\Gamma\left(\pi_{E}\right)$ by or $_{E, A}$. Consider the decomposition $\left.\pi\right|_{E}=\pi_{b} \oplus \pi_{c}$ into a direct sum of two line sub-bundles of degrees $b$ and $c$, respectively, $b \geqslant c \geqslant 0$.

A section of $\pi_{A}$ is the sum of a section $\sigma_{b}$ of $\pi_{b}$ and a section $\sigma_{c}$ of $\pi_{c}$. Note that the degree $a$ is equal to the number of common zeros of $\sigma_{b}$ and $\sigma_{c}$ (counted with multiplicities). Thus, varying $\sigma_{b}$ and $\sigma_{c}$, we get a deformation of the line sub-bundle $\pi_{A}$ within the line sub-bundles of the same degree, as long as the common zeros are deformed together, and no new common zeros are introduced (even if, at some instant of the deformation, a zero of $\sigma_{b}$ or $\sigma_{c}$ passes through one of the common zeros). The orientation $\operatorname{or}_{E, A}$ is unchanged under such a deformation.

Consider the circle bundle $F_{+} \rightarrow \mathbb{R} K$ obtained by positive projectivization of $\pi_{E}$. Here $F_{+}$is a (topological) surface obtained from $\mathbb{R} E$ by removing the zero section of $\pi_{E}$ and identifying the positive scalar multiples in the same fiber of $\pi_{E}$. Depending on the parity of $d=b+c$, the surface $F_{+}$is a torus or a Klein bottle. We have $H_{1}\left(F_{+} ; \mathbb{Z}_{2}\right) \approx \mathbb{Z}_{2}^{2}$, in particular the subset $\mathcal{S} \subset H_{1}\left(F_{+} ; \mathbb{Z}_{2}\right)$ consisting of the classes with non-trivial image under the homomorphism induced by $F_{+} \rightarrow \mathbb{R} K$ is a 2 -element set.

Given an oriented line sub-bundle $\pi_{A} \subset \pi_{E}$, denote by $s(A) \in \mathcal{S}$ the homology class of the projectivizations of the positively-directed vectors in $\pi_{A}$. Given a non-orientable line sub-bundle $\pi_{A} \subset \pi_{E}$ ( $a$ is odd) and a local orientation of $\pi_{E}$ over $x_{0}$, we may add (topologically) a left half-twist to the sub-bundle $\pi_{A}$ to obtain an orientable bundle and proceed as above to define $s(A) \in \mathcal{S}$. Note that, in the case where both $a$ and $d$ are odd, and the orientation of $\pi_{E / A}$ is fixed (in particular, over $x_{0}$ ), then $s(A)$ depends only on this orientation, as a different choice of the local orientation of $\pi_{E}$ over $x_{0}$ changes both the direction of the half-twist and the orientation of the line bundle after the halftwist. Thus, the class $s(A)$ is well defined once we fix the same orientation data as in Corollary 6.6, i.e. an orientation of one of $\pi_{A}, \pi_{E / A}$ and $\pi_{E}$, depending on the parity of $d$ and $a$. Furthermore, a different choice of this orientation results in the different value for $s(A)$.

LEMma 6.7. Let $\pi_{A_{0}}$ and $\pi_{A_{1}}$ be two line sub-bundles of $\pi_{E}$ of the same degree $a \geqslant 0$.

We have or $_{E, A_{0}}=\mathrm{or}_{E, A_{1}}$ if and only if $s\left(A_{0}\right)=s\left(A_{1}\right)$.

Proof. Consider generic sections $\xi_{A_{0}}$ and $\xi_{A_{1}}$ of $\pi_{A_{0}}$ and $\pi_{A_{1}}$, respectively, with $a$ distinct real zeros each. These sections can be deformed into each other in the class of sections of $\pi_{E}$ with $a$ distinct real zeros. This deformation may be assumed generic. Each intermediate section defines a line sub-bundle $\pi_{A_{t}} \subset \pi_{E}$, and can be decomposed into the summands $\sigma_{b_{t}}$ and $\sigma_{c_{t}}, t \in[0,1]$. If the zeros of $\sigma_{b_{t}}$ and $\sigma_{c_{t}}$ do not collide, then 
both or $\left(E, A_{t}\right)$ and $s\left(A_{t}\right)$ stay invariant.

Suppose that two simple zeros of $\sigma_{b_{t}}$ and $\sigma_{c_{t}}$ collide at $t=t_{0} \in[0,1]$ in a point $y \in \mathbb{R} K$ so that the orders of these zeros for $t_{0}-\varepsilon$ and $t_{0}+\varepsilon$ get reversed. Pass to the fiberwise projectivization of $\pi_{E}$ (which is a Hirzebruch surface $F_{b-c}$ ). The projectivization of $\pi_{A_{t}}$ corresponds to a curve intersecting a generic fiber once. At $t=t_{0}$ this curve degenerates to the union of the fiber $F_{y}$ over $y$ and the residue curve intersecting $F_{y}$ transversally. The zeros of a section of $\pi_{E / A}$ correspond to the intersection points of this curve with another curve resulting from a section of $\pi_{E}$. This curve intersects $F_{y}$ once. For $t=t_{0} \pm \varepsilon$ the fiber $F_{y}$ gets added to the residue curve with different orientation, so that the orientations of $\pi_{E / A_{t-\varepsilon}}$ and $\pi_{E / A_{t+\varepsilon}}$ given by Lemma 6.5 are opposite. The classes $s\left(A_{t-\varepsilon}\right)$ and $s\left(A_{t+\varepsilon}\right)$ are also different, as the corresponding projectivization are different by the fiber of $F_{+}$ over $y$.

Corollary 6.8. Let $\pi_{A}$ and $\pi_{B}$ be line sub-bundles of $\pi_{E}$ of degrees $a \geqslant 0$ and $b \geqslant 0$, respectively. There exists a sign $\sigma(a, b)= \pm 1$ depending only on the numbers $a$ and $b$ such that or $_{E, A}=\sigma(a, b)$ or $_{E, B}$ if and only if $s(A)=s(B)$.

Proof. Define $\sigma(a, b)$ so that the equality $\operatorname{or}_{E, A}=\sigma(a, b) \operatorname{or}_{E, B}$ holds for two particular line sub-bundles $\pi_{A}$ and $\pi_{B}$ of degree $a$ and $b$, respectively. This sign depends only on $a$ and $b$, by Lemma 6.7 .

Remark 6.9. Note that a Spin-structure on an orientable 2-dimensional vector bundle $\pi_{E}$ is a choice of an orientation of $\pi_{E}$ together with a bijective map $\mathcal{S} \rightarrow\{ \pm 1\}$. If $\pi_{E}$ is non-orientable, a choice of bijection $\mathcal{S} \rightarrow\{ \pm 1\}$ is known as a Pin_-structure. (This interpretation of Spin and Pin_-structures holds for any real rank-2 vector bundle over a topological circle.)

Thus, Lemma 6.7 simply states that, for a given $a$, the orientation of $\Gamma\left(\pi_{E}\right)$ is determined by the Spin- or Pin_-structure on $\pi_{E}$ as long as a sub-bundle $\pi_{A}$ of degree $a$ in $\pi_{E}$ exists. Namely, we take or $_{E, A}$ if the value of Spin/Pin_-structure on $s(A)$ is positive, and - or $_{E, A}$ otherwise. If $\mathbb{R} K=\mathbb{R} \widetilde{C}$ and $\pi_{E}$ is the pull-back of the tangent bundle, then the Spin/Pin_-structure is given by the logarithmic trivialization of $\left(\mathbb{C}^{\times}\right)^{2} \subset \mathbb{C} \Delta$. This connection can be traced in the proof below (even though the proof does not use it explicitly).

Proof of Proposition 6.4. Consider the connected component $\mathcal{C}$. The tangent vector fields

$$
(z, w) \longmapsto z \frac{\partial}{\partial z} \quad \text { and } \quad(z, w) \longmapsto w \frac{\partial}{\partial w}
$$

on $\left(\mathbb{C}^{\times}\right)^{2}$ are non-vanishing, and extend to (possibly vanishing on $\partial \mathbb{C} \Delta$ ) holomorphic vector fields on $\mathbb{C} \Delta$ away from a finite number of intersections of toric divisors in $\mathbb{C} \Delta$. In 
particular, for $\mathbb{R} C^{\circ} \in \mathcal{C}$, these vector fields provide sections for the pull-back $\pi_{E^{C}}$ of the tangent bundles $T \mathbb{C} \Delta$ to the normalization $\mathbb{R} \widetilde{C}$ of $\mathbb{R} \bar{C}$. Therefore, the bundles $\pi_{E^{C}}$ are generated by global sections. Furthermore, one of these vector fields, say

$$
z \frac{\partial}{\partial z}
$$

defines a line sub-bundle $\pi_{A^{C}} \subset \pi_{E^{C}}$ well defined for all $\mathbb{R} C^{\circ} \in \mathcal{M}_{\Delta}$.

The number of positive (adjacent to the positive quadrant $\left(\mathbb{R}_{>0}\right)^{2}$ ) and negative points of $\mathcal{P}_{t} \subset \partial \mathbb{R} \Delta$ contained in a divisor $\mathbb{R} E \subset \partial \mathbb{R} \Delta$ is determined by $\lambda$ and is independent of $t$. If $\mathbb{R} E \cap \partial\left(\mathbb{R}_{>0}\right)^{2} \cap \mathcal{P}_{t} \neq \varnothing$, then we choose a reference point

$$
p_{t}^{+} \in \mathbb{R} E \cap \mathcal{P}_{t}
$$

as the first point on $\mathbb{R} E \cap \partial\left(\mathbb{R}_{>0}\right)^{2}$ in the linear order induced by the clockwise orientation of $\partial\left(\mathbb{R}_{>0}\right)^{2}$. Note that, by the definition of $\mathcal{M}_{\Delta}$, the $m$ points of $\mathcal{P}_{t}$ are always distinct. Thus, $p_{+} \in \mathbb{R} \bar{C}$ is a consistent choice of a reference point for $\mathbb{R} C^{\circ} \in \mathcal{M}_{\Delta}$.

Furthermore, at $p_{+}$we have a canonical choice of a local orientation of $\mathbb{R} \Delta$ (coming from the standard orientation of $\mathbb{R}_{>0}^{2}$ ). The orientations of $\pi_{A^{C}}$ and $\pi_{E^{C}} / \pi_{A^{C}}$ in the positive quadrant, and thus at $p_{+}$, are also standard. Corollary 6.6 provides an orientation of the bundle over $\mathcal{M}_{\Delta} \ni \mathbb{R} C^{\circ}$ formed by $\Gamma\left(\pi_{E^{C}}\right)$, the space of sections of the 2-dimensional bundle $\pi_{E^{C}}$ over the normalization $\mathbb{R} \widetilde{C}$.

The tangent space to $\mathcal{M}_{\Delta}$ at $\mathbb{R} C^{\circ}$ can be identified with the quotient of the vector space $\Gamma\left(\pi_{E^{C}}\right)$ by its 3 -dimensional subspace $\Gamma_{B}$ obtained as the image of the tangent vector fields on $\mathbb{R} \widetilde{C}$ in the tangent bundle of $\mathbb{R} \Delta$. In the case where $\mathbb{R} \bar{C}$ is immersed, we have $\Gamma_{B}=\Gamma\left(\pi_{E^{B}}\right)$, where $\pi_{E_{B}} \subset \pi_{E^{C}}$ is the tangent bundle to $\mathbb{R} \widetilde{C}$. The orientation of $\Gamma_{B}$ is provided by Lemma 6.5. The orientation of $\mathcal{M}_{\Delta}$ (i.e. the orientation of its tangent bundle) is consistently determined by this quotient.

If no points of $\mathbb{R} \bar{C} \cap \partial \mathbb{R} \Delta$ are adjacent to the positive quadrant, then $\mathbb{R} \widetilde{C}$ is mapped to the same quadrant by the normalization map $\mathbb{R} \widetilde{C} \rightarrow \mathbb{R} \bar{C}$. The standard orientation of this quadrant induces a consistent orientation of the bundle $\pi_{E^{C}}$ which has an even degree in this case. Thus, Corollary 6.6 provides (once again, through the quotient by $\Gamma_{B}$ ) an orientation of the corresponding components of $\mathcal{M}_{\Delta}$ in this case as well.

Recall that the space $\mathcal{M}_{\partial \Delta}$ consists of the conj-invariant $m$-tuples of distinct points in $\partial \mathbb{C} \Delta$. Thus, it is oriented by the standard (clockwise with respect to the positive real quadrant) orientations of real toric divisors and the order of points on these divisors corresponding to these orientations. Indeed, this yields an order for all real points of the $m$-tuple. Each conjugate pair of imaginary points is contained in $\partial \mathbb{C} \Delta \backslash \partial \mathbb{R} \Delta$. Exactly one point in each pair is contained in a connected component $E^{+} \subset \partial \mathbb{C} \Delta \backslash \partial \mathbb{R} \Delta$ whose 
complex orientation agrees with the chosen orientation on $\partial \mathbb{R} \Delta$. The conjugate pair is determined by this representative point. The orientation of $\mathcal{M}_{\Delta}$ comes as the product of the orientations of $\partial \mathbb{R} \Delta$ (in the induced order of the real points of the $m$-tuple) and the complex orientations of $\partial \mathbb{C} \Delta$ at the representative points of the conjugate pairs. Note that the relative order of conjugate pairs is irrelevant for the product orientation.

Thus, the map (6.8) is a map between oriented manifolds. To prove (6.9), it suffices to show that the local degree of the map (6.8) at $\mathbb{R} C^{\circ} \in \mathcal{M}_{\Delta}$ agrees with $\sigma(\mathbb{R} C$ ) (up to a global sign on $\mathcal{C})$ in the case where $\operatorname{ev}\left(\mathbb{R} C^{\circ}\right)$ is a regular value.

Note that the tangent space $T_{\operatorname{ev}\left(\mathbb{R} C^{\circ}\right)} \mathcal{M}_{\partial \Delta}$ is identified with the tangent space $T_{\mathbb{R} C^{\circ}} \mathcal{M}_{\Delta}$ by the differential of the map (6.8) at its regular point $\mathbb{R} C^{\circ}$. We get

$$
T_{\mathrm{ev}\left(\mathbb{R} C^{\circ}\right)} \mathcal{M}_{\partial \Delta}=T_{\mathbb{R} C^{\circ}} \mathcal{M}_{\Delta}=\Gamma\left(\pi_{E^{C}} / \pi_{B^{C}}\right) .
$$

The orientation of $\Gamma\left(\pi_{E^{C}} / \pi_{B^{C}}\right)$ given by Lemma 6.5 differs from the orientation of $T_{\mathrm{ev}\left(\mathbb{R} C^{\circ}\right)} \mathcal{M}_{\partial \Delta}$ according to the cyclic order of $\mathbb{R} \widetilde{C} \cap \partial \mathbb{R} \Delta$ at $\mathbb{R} \widetilde{C}$. Thus, these orientations agree or disagree uniformly on $\mathcal{C}$.

To compare the orientations of $T_{\mathbb{R} C^{\circ}} \mathcal{M}_{\Delta}$ and $T_{\mathrm{ev}\left(\mathbb{R} C^{\circ}\right)} \mathcal{M}_{\partial \Delta}$, we use the identification (6.12). Each of these orientations corresponds to an orientation of the vector space $\Gamma\left(\pi_{E^{C}}\right)$ induced by the presentation $\Gamma\left(\pi_{E^{C}} / \pi_{B^{C}}\right)=\Gamma\left(\pi_{E^{C}}\right) / \Gamma\left(\pi_{B^{C}}\right)$ (which holds in the case where $\mathbb{R} \bar{C}$ is immersed in $\mathbb{R} \Delta$ ). One orientation is defined by the sub-bundle $\pi_{E^{A}} \subset$ $\pi_{E^{C}}$, the other one by $\pi_{B^{C}} \subset \pi_{E^{C}}$. By Corollary 6.8 , these orientations agree or not, depending on the values $s\left(E^{A}\right), s\left(E^{B}\right) \in \mathcal{S}$.

Note that the pull-backs of the tangent bundle of $\mathbb{R} \Delta$ to $\mathbb{R} \widetilde{C}$ are canonically isomorphic as vector bundles over $\mathbb{R} \widetilde{C}$ (treated as a topological space) for all $\mathbb{R} C^{\circ} \in \mathcal{C}$. Thus, we may identify the 2 -element sets $\mathcal{S}$ for different curves in $\mathcal{C}$. By the definition of $\pi_{A^{C}}$, the elements $s\left(E^{A}\right)$ agree under this identification. At the same time, the value of $s\left(E^{B}\right)$ depends on $\operatorname{Rot}_{\mathrm{Log}}(\mathbb{R} C)$. As each half-turn at the positive projectivization $F_{+}$of $\pi_{E}^{C}$ corresponds to a full turn in the projectivization of $\pi_{E^{C}}$, the value of $s\left(B^{C}\right) \in \mathcal{S} \subset H_{1}\left(F_{+} ; \mathbb{Z}_{2}\right)$ is determined by the mod- 4 residue of $\operatorname{Rot}_{\log }(\mathbb{R} C)$, and thus by $\sigma(\mathbb{R} C)$.

\subsection{Proof of Theorems 5.5 and 5.7}

Proof of Theorem 5.5. In the previous subsection we have proved the invariance of $R_{\Delta, k}(\mathcal{P})$ under continuous deformations of $\mathcal{P} \in \mathcal{M}_{\partial \Delta}$ disjoint from the image of the locus of reducible curves in $\mathcal{M}_{\Delta}$ under ev. Suppose now that

$$
\mathcal{R}_{\Delta}\left(\mathcal{P}_{t}\right)=\bigcup_{k^{\prime}=-\operatorname{Area}(\Delta)}^{\operatorname{Area}(\Delta)} \mathcal{R}_{\Delta, k^{\prime}}\left(\mathcal{P}_{t}\right)
$$


contains a reducible curve $\mathbb{R} \bar{D}$ for $t=t_{0}$.

As the dimension of the space of deformations of each component is equal to the number of points in its intersection with $\partial \mathbb{C} \Delta$ minus 1 , for the generic path $\gamma$ (in the nonsingular space $\left.\mathcal{M}_{\Delta}\right)$ the curve $\mathrm{Sq}^{\Delta}(\mathbb{C} \bar{D})$ is the union of two irreducible rational immersed curves $\mathrm{Sq}^{\Delta}\left(\mathbb{C} \bar{D}_{j}\right), j=1,2$. These two curves define a subdivision of the configuration $\mathcal{P}_{t_{0}}$ (which is no longer generic in $\mathcal{M}_{\Delta}$ ) into the disjoint union of two real generic configurations $\mathcal{P}_{t_{0}}^{(j)}=\mathrm{Sq}^{\Delta}\left(\mathbb{C} \bar{D}_{j}\right) \cap \partial \Delta, j=1,2$, which can be presented as squares of the Menelaus configurations $\mathcal{Q}_{t}^{(j)}=\mathbb{C} \bar{D}_{j} \cap \partial \Delta$. Thus, each curve $\mathbb{C} \bar{D}_{j}$ is defined over $\mathbb{R}$. Moreover, the presence of multiplicative translations in $\left(\mathbb{R}^{\times}\right)^{2}$ implies that $\mathrm{Sq}^{\Delta}\left(\mathbb{R} \bar{D}_{1}\right)$ and $\mathrm{Sq}^{\Delta}\left(\mathbb{R} \bar{D}_{2}\right)$ intersect transversally.

We may assume that all points of $\mathcal{P}_{t}$, except for two points $p_{j}(t) \in \mathcal{P}_{t}, j=1,2$, remain independent of $t \in\left[t_{0}-\varepsilon, t_{0}+\varepsilon\right]$ for a small $\varepsilon>0$. For $t \in\left[t_{0}-\varepsilon, t_{0}+\varepsilon\right]$ the deformation $p_{1}(t) \in \partial \mathbb{R} \Delta$ of $p_{1}\left(t_{0}\right)$ determines the deformation $p_{2}(t)$ of $p_{2}\left(t_{0}\right)$, as $\mathcal{P}_{t}$ is the square of a Menelaus configuration for all $t$. The points $p_{1}\left(t_{0}\right)$ and $p_{2}\left(t_{0}\right)$ must belong to two different components of $\mathbb{R} \bar{D}$. Indeed, otherwise one of the components of $\mathbb{R} \bar{D}$ contains only points of $\mathcal{P}_{t}$ independent of $t$, and then $\mathcal{R}_{\Delta}\left(\mathcal{P}_{t}\right)$ contains reducible curves for all $t \in\left[t_{0}-\varepsilon, t_{0}+\varepsilon\right]$, which contradicts to our assumption. For $t_{0}-\varepsilon \leqslant t \leqslant t_{0}+\varepsilon$, denote by $\mathcal{R}_{\Delta}^{\mathbb{R} D}\left(\mathcal{P}_{t}\right)$ the curves whose images under $\mathrm{Sq}^{\Delta}$ is close to $\mathrm{Sq}^{\Delta}(\mathbb{R} \bar{D})$.

Then, the intersection points of

$$
I=\mathrm{Sq}^{\Delta}\left(\mathbb{R} \bar{D}_{1}\right) \cap \mathrm{Sq}^{\Delta}\left(\mathbb{R} \bar{D}_{2}\right)
$$

come with the intersection sign in $\mathbb{R}_{>0}^{2}$. The set of positive points $I_{+} \subset I$ has the same cardinality as the set of negative points $I_{-} \subset I$, as $\mathbb{R}_{>0}^{2}$ is contractible.

The curves in $\mathcal{R}_{\Delta}^{\mathbb{R} D}\left(\mathcal{P}_{t_{0} \pm \varepsilon}\right)$ are obtained by smoothing a nodal point $q \in I$ in one of the two ways, one that agrees with our choice of orientation and one that does not. Without loss of generality (changing the direction of the path $\gamma$, if needed) we may assume that the orientation-preserving smoothing in a point $q \in I_{+}$corresponds to a curve $\mathbb{R} \bar{D}_{q,+} \in$ $\mathcal{R}_{\Delta}\left(\mathcal{P}_{t_{0}+\varepsilon}\right)$, and thus the orientation-reversing smoothing at the same point corresponds to a curve $\mathbb{R} \bar{D}_{q,-} \in \mathcal{R}_{\Delta}\left(\mathcal{P}_{t_{0}-\varepsilon}\right)$. The following lemma determines the situation at all the other points of $I$.

LEMma 6.10. A curve obtained by the smoothing of a node from $I_{+}$in the orientationpreserving way, or by the smoothing of a node from $I_{-}$in the orientation-reversing way, belongs to $\mathcal{R}_{\Delta}\left(\mathcal{P}_{t_{0}+\varepsilon}\right), \varepsilon>0$.

Accordingly, a curve obtained by the smoothing of a node from $I_{-}$in the orientationpreserving way, or by the smoothing of a node from $I_{+}$in the orientation-reversing way, belongs to $\mathcal{R}_{\Delta}\left(\mathcal{P}_{t_{0}-\varepsilon}\right)$. 

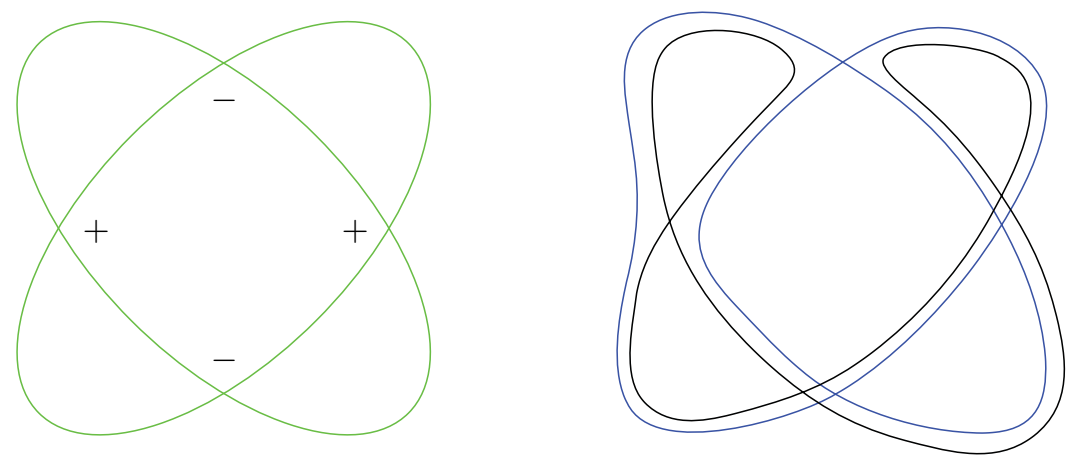

Figure 6.1. The signs of intersection points of two components of $\mathbb{R} D$ and the corresponding direction of smoothing.

Proof. Let $\mathbb{R} D_{q^{\prime}, s} \in \mathcal{R}_{\Delta}\left(\mathcal{P}_{t_{0}+\varepsilon}\right), s= \pm 1$, be the curve obtained by smoothing $\mathbb{R} D$ at a point $q^{\prime} \in I$ according to the sign $s$. Note that $\mathrm{Sq}^{\Delta}\left(\mathbb{C} D_{q,+}\right)$ and $\mathrm{Sq}^{\Delta}\left(\mathbb{C} D_{q^{\prime}, s}\right)$ are tangent to each other at $m$ points of $\mathcal{P}_{t_{0}+\varepsilon}$ and must intersect each other at pairs of points close to each point of $I^{\mathbb{C}}=\mathrm{Sq}^{\Delta}\left(\mathbb{C} \bar{D}_{1}\right) \cap \mathrm{Sq}^{\Delta}\left(\mathbb{C} \bar{D}_{2}\right)$, except for the smoothing points $q$ and $q^{\prime}$. Tangencies contribute at least $2 m$ to the intersection of $\mathrm{Sq}^{\Delta}\left(\mathbb{C} D_{q,+}\right)$ and $\mathrm{Sq}^{\Delta}\left(\mathbb{C} D_{q^{\prime}, s}\right)$, this number being the integer perimeter of the polygon $2 \Delta$. The cardinality of the set $I^{\mathbb{C}} \backslash\left\{q, q^{\prime}\right\}$ is one less than the number of lattice points inside the polygon $2 \Delta$ by the genus formula, since $\mathrm{Sq}^{\Delta}\left(\mathbb{C} D_{q,+}\right)$ is a rational curve and $2 \Delta$ is its Newton polygon. Thus, by Pick's formula, we have already identified $2 \operatorname{Area}(2 \Delta)$ points in

$$
\mathrm{Sq}^{\Delta}\left(\mathbb{C} D_{q,+}\right) \cap \mathrm{Sq}^{\Delta}\left(\mathbb{C} D_{q^{\prime}, s}\right)
$$

counting with multiplicities. By the Kushnirenko-Bernstein formula, the curves

$$
\mathrm{Sq}^{\Delta}\left(\mathbb{R} D_{q,+}\right) \quad \text { and } \quad \mathrm{Sq}^{\Delta}\left(\mathbb{R} D_{q^{\prime}, s}\right)
$$

may not have any other intersection points, and this fact implies that $s=1$ if $q^{\prime} \in I_{+}$; see Figure 6.1.

Note that any curve in $\mathcal{R}_{\Delta}^{\mathbb{R} D}\left(\mathcal{P}_{t_{0} \pm \varepsilon}\right)$ is obtained by smoothing $\mathbb{R} D$ at a point $q \in I$. The quantum index of the result is $\pm k\left(\mathbb{R} D_{1}\right) \pm k\left(\mathbb{R} D_{2}\right)$, where the signs are determined by the agreement or disagreement of the orientation of the resulting curve with the chosen orientations of $\mathbb{R} D_{j}$. Since $\# I_{+}=\# I_{-}$, Lemma 6.10 implies that $\mathcal{R}_{\Delta}^{\mathbb{R} D}\left(\mathcal{P}_{t_{0}+\varepsilon}\right)$ and $\mathcal{R}_{\Delta}^{\mathbb{R} D}\left(\mathcal{P}_{t_{0}-\varepsilon}\right)$ have the same number of curves of each quantum index.

Proof of Theorem 5.7. By Proposition 6.4, we have $\widetilde{R}_{\Delta_{d}, k}(\mathcal{P})=\widetilde{R}_{\Delta_{d}, k}\left(\mathcal{P}^{\prime}\right)$ if there are no reducible curves with Newton polygon $\Delta_{d}$ that pass through $\mathcal{P}_{t}$. Also, we may assume that, if $\mathbb{R} D$ is a reducible curve with Newton polygon $\Delta_{d}$ passing through $\mathcal{P}_{t_{0}}$, 
then it consists of two components $\mathbb{R} D_{1}$ and $\mathbb{R} D_{2}$ that intersect transversely at a finite set $I$. Note that the degree of both components, $\mathbb{R} D_{1}$ and $\mathbb{R} D_{2}$, must be even, as a real curve of odd degree must intersect $\partial \mathbb{R P}^{2}$ in a negative point, as the boundary of the positive quadrant is null-homologous.

We have two smoothings of $\mathbb{R} D$ at $q \in I$ that pass through $\mathcal{P}_{t_{0}-\varepsilon}$ and $\mathcal{P}_{t_{0}+\varepsilon}$. One can be oriented in accordance with the orientations of $\mathbb{R} D_{1}$ and $\mathbb{R} D_{2}$, and the other one in accordance with the orientation of $\mathbb{R} D_{1}$, but opposite to the orientation of $\mathbb{R} D_{2}$. The corresponding quantum indices are different by $2 k\left(\mathbb{R} D_{2}\right)$. The index $k\left(\mathbb{R} D_{2}\right)$ is integer, since the degree of $\mathbb{R} D_{2}$ is even.

\subsection{Indices of real phase-tropical curves}

We start by recalling the basic notions of tropical geometry (cf. [18], [19]), specializing to the case of plane curves. Recall that a metric graph is a topological space homeomorphic to $\Gamma^{\circ}=\Gamma \backslash \partial \Gamma$ enhanced with a complete inner metric. Here, $\Gamma$ is a finite graph and $\partial \Gamma$ is the set of its 1-valent vertices. The metric graph is also sometimes called a tropical curve (while in some other instances the term tropical curve is reserved for the equivalence class of metric graphs with respect to tropical modifications). In this paper, we require the graph $\Gamma$ to be connected, so that $\Gamma^{\circ}$ is irreducible as a tropical curve. We assume that $\Gamma$ has a vertex of valence at least 3 , and that $\Gamma$ does not have 2 -valent vertices. The half-open edges of $\Gamma^{\circ}$ obtained from the closed edges of $\Gamma$ adjacent to $\partial \Gamma$ are called leaves.

A plane tropical curve is a proper continuous map $h: \Gamma^{\circ} \rightarrow \mathbb{R}^{2}$ such that $\left.h\right|_{E}$ is smooth for every edge $E \subset \Gamma$ with $d h(u) \in \mathbb{Z}^{2}$ for a unit tangent vector $u$ at any point of $E$. In addition, we require the following balancing condition at every vertex $v \in \Gamma$ :

$$
\sum_{E} d h(u(E))=0
$$

where $u(E)$ is the unit tangent vector in the outgoing direction with respect to $v$, and the sum is taken over all edges $E$ adjacent to $v$.

The collection of vectors $\left\{d h\left(u_{v}\right)\right\}_{v \in \partial \Gamma}$, where $u_{v}$ is a unit vector tangent to the leaf adjacent to $v$ (and directed away from $v$ ), is called the (toric) degree of $h: \Gamma^{\circ} \rightarrow \mathbb{R}^{2}$. The identity (6.15) implies that the sum of all vectors in this collection is zero. Therefore, this collection is dual to a lattice polygon $\Delta \in \mathbb{Z}^{2}$, which is well defined up to translations in $\mathbb{Z}^{2}$. The polygon $\Delta$ is determined by $h\left(\Gamma^{\circ}\right)$. We call $\Delta$ the Newton polygon of $h: \Gamma^{\circ} \rightarrow \mathbb{R}^{2}$.

Tropical curves appear as limits of scaled sequences of complex curves in the plane. Let $A$ be any set and $\alpha \mapsto t_{\alpha} \in \mathbb{R}$ be a function unbounded from above (this function is 
called the tropical scaling sequence). Let $\mathbb{C} C_{\alpha} \subset\left(\mathbb{C}^{\times}\right)^{2}, \alpha \in A$, be a family of complex curves with Newton polygon $\Delta$.

Definition 6.11. We say that a family $\mathbb{C} C_{\alpha}$ has a phase-tropical limit with respect to $t_{\alpha}$ if for every $p \in \mathbb{R}^{2}$ we have

$$
\lim _{t_{\alpha} \rightarrow \infty} t_{\alpha}^{-p} \mathbb{C} C_{\alpha}=\Phi_{p}
$$

for a (possibly empty) algebraic curve $\Phi(p) \subset\left(\mathbb{C}^{\times}\right)^{2}$. Here $t_{\alpha}^{-p} \mathbb{C} C_{\alpha}$ is the multiplicative translation of the curve $\mathbb{C} C_{\alpha}$ by $t_{\alpha}^{-p} \in\left(\mathbb{C}^{\times}\right)^{2}$. The coefficients of the polynomials defining $t_{\alpha}^{-p} \mathbb{C} C_{\alpha}$ represent a point in the projective space of dimension $\#\left(\Delta \cap \mathbb{Z}^{2}\right)-1$. The limit is understood in the sense of the topology of this projective space. The curve $\Phi_{p} \subset\left(\mathbb{C}^{\times}\right)^{2}$ may be reducible and even non-reduced.

We say that $h: \Gamma^{\circ} \rightarrow \mathbb{R}^{2}$ is the tropical limit of $\mathbb{C} C_{\alpha}$ with respect to $t_{\alpha}$ if, for a sufficiently small open convex neighborhood $U \subset \mathbb{R}^{2}$ of $p$, the irreducible components $\Psi \subset \Phi(p) \subset\left(\mathbb{C}^{\times}\right)^{2}$ correspond to the connected components $\psi \subset h^{-1}(U)$, so that the lattice polygon $\Delta_{\psi}$ determined by the ends of the open graph $\psi$ coincides with the Newton polygon $\Delta_{\Psi}$ of the irreducible component $\Psi$ taken with some multiplicity. The same component $\Psi$ may correspond to several components of $h^{-1}(U)$, so that the sum of all resulting multiplicities is equal to the multiplicity of $\Psi$ in $\Phi(p)$. Each connected component of $h^{-1}(U)$ corresponds to a unique component of $\Phi(p)$.

If $h$ does not contract any edge of $\Gamma^{\circ}$ to a point, then the open set $\psi \subset \Gamma^{\circ}$ may contain at most one vertex. If $v \in \Gamma$ is such a vertex, then we call $\Psi$ the phase $\Phi_{v}$ of the vertex $v$. If $\psi$ is contained in an edge $E$, then we call $\Psi$ the phase $\Phi_{E}$ of the edge $E$. The phases $\Phi(E) \subset\left(\mathbb{C}^{\times}\right)^{2}$ do not depend on the choice of the point $p \in h(E)$, and are well defined up to multiplicative translations by $\left(\mathbb{R}^{\times}\right)^{2}$. The curve $h: \Gamma^{\circ} \rightarrow \mathbb{R}^{2}$ enhanced with the phases $\Phi_{v}$ and $\Phi_{E}$ for its vertices and edges is called the phase-tropical limit of $\mathbb{C} C_{\alpha}$ with respect to the scaling sequence $t_{\alpha} \rightarrow \infty$.

We consider equivalent phases in $\left(\mathbb{C}^{\times}\right)^{2}$ that are different by multiplicative translations by vectors from $\left(\mathbb{R}_{>0}\right)^{2}$.

Note that the Newton polygon of the phase $\Phi_{E}$ of an edge $E$ is an interval. Thus, after a suitable change of coordinates in $\left(\mathbb{C}^{\times}\right)^{2}$, the (irreducible) curve $\Phi_{E}$ is given by a linear equation in one variable. Therefore, $\Phi_{E}$ is a multiplicative translation of a subtorus $S^{1} \approx T_{E} \subset S^{1} \times S^{1}$ in the direction parallel to $h(E)$.

Let us orient $E$. Then $T_{E}$, as well as the quotient space $B_{E}=\left(S^{1} \times S^{1}\right) / T_{E}$, also acquire an orientation. The image $\operatorname{Arg}\left(\Phi_{E}\right)$ coincides with $\pi_{E}^{-1}\left(\sigma_{E}\right)$ for some $\sigma_{E} \in B_{E}$, where $\pi_{E}: S^{1} \times S^{1} \rightarrow B_{E}$ is the projection. Since $B_{E}$ is isomorphic to $S^{1}$ and oriented, we have a canonical isomorphism $B_{E}=\mathbb{Z} / 2 \pi \mathbb{Z}$. Thus, a phase $\Phi_{E}$ of an oriented edge $E$ of 
a planar tropical curve is determined by a single argument $\sigma(E) \in \mathbb{Z} / 2 \pi \mathbb{Z}$. The change of orientation of $E$ results in the change of sign of $\sigma(E)$.

Let $v \in \Gamma^{\circ}$ be a vertex and $E_{j}$ be the edges adjacent to $v$. Orient $E_{j}$ outwards from $v$. With the oriented edges $E_{j}$ can be associated a momentum $\mu\left(E_{j}\right)$ with respect to the origin $0 \in \mathbb{R}^{2}$. This is the wedge product of the vector connecting the origin with a point of $E_{j}$ and the unit tangent vector $u\left(E_{j}\right)$ coherent with the orientation. Clearly, it does not depend on the choice of the point in $E_{j}$.

Recall that the vertex $v$ is dual to the lattice polygon $\Delta_{v}$ determined by the integer vectors $d h\left(u\left(E_{j}\right)\right)$. The multiplicity is defined as $m(v)=2 \operatorname{Area}\left(\Delta_{v}\right)$; cf. [18].

Proposition 6.12. (tropical Menelaus theorem) For any tropical curve $h: \Gamma^{\circ} \rightarrow \mathbb{R}^{2}$ and a vertex $v \in \Gamma^{\circ}$ the momenta $\mu\left(E_{j}\right)$ of the edges adjacent to $v$ and oriented outwards from $v$ satisfy to the equality

$$
\sum_{j} \mu\left(E_{j}\right)=0
$$

If $\sigma\left(E_{j}\right) \in \mathbb{Z} / 2 \pi \mathbb{Z}$ are phases of the oriented edges $E_{j}$, then

$$
\sum_{j} w\left(E_{j}\right) \sigma\left(E_{j}\right)=\pi m(v)
$$

(assuming that $\sigma\left(E_{j}\right)$ appear in the phase-tropical limit of a family $\mathbb{C} C_{\alpha} \subset\left(\mathbb{C}^{\times}\right)^{2}$ of complex curves).

This statement can be viewed as a counterpart of the ancient Menelaus theorem (before its generalizations by Carnot and Weil) stating that three points $D, E$ and $F$ on the extensions of three sides of a planar triangle $A B C$ are collinear if and only if

$$
\frac{|A D|}{|D B|} \frac{|B E|}{|E C|} \frac{|C F|}{|F A|}=-1 .
$$

Here, the length is taken with the negative sign if the direction of an interval (e.g. $|C F|$ ) is opposite to the orientation of the triangle; see Figure 6.2.

Proof. The wedge product of the balancing condition (6.15) with the vector connecting 0 and $v$ gives (6.17). To deduce (6.18), we consider the polynomial $f_{v}$ (whose Newton polygon is $\Delta_{v}$ ) defining the phase $\Phi_{v} \subset\left(\mathbb{C}^{\times}\right)^{2}$. By Vieta's theorem, the product of the roots cut by $f_{v}$ on a divisor of $\mathbb{C} \Delta_{v}$ corresponding to an oriented side $F \subset \Delta_{v}$ is $(-1)^{\#\left(F \cap \mathbb{Z}^{2}\right)}$ times the ratio of the coefficients at the endpoints of $F$. Therefore, the sum of the phases of the edges of $\Gamma$ corresponding to $F$ is the argument of this ratio plus $\#\left(F \cap \mathbb{Z}^{2}\right) \pi$. Since by Pick's formula the parity of $\#\left(\partial \Delta \cap \mathbb{Z}^{2}\right)$ coincides with that of $m(v)=2 \operatorname{Area}\left(\Delta_{v}\right)$, we recover (6.18). 




Figure 6.2. The Menelaus theorem.

COROLlaRY 6.13. We have $\sum_{E} \mu(E)=0$, where the sum is taken over all leaves of $h: \Gamma^{\circ} \rightarrow \mathbb{R}^{2}$ oriented in the outwards direction.

Proof. Take the sum of the expression (6.17) over all vertices of $\Gamma^{\circ}$. The momenta of all bounded edges will enter twice with opposite signs.

If all curves $\mathbb{C} C_{\alpha}$ are defined over $\mathbb{R}$, then the phases $\Phi(p)$ must be real for all points $p \in \mathbb{R}^{2}$. Note, however, that in general the phase $\Phi_{v}$ for a vertex $v \in \Gamma^{\circ}$ does not have to be real, as the involution of complex conjugation may exchange it with $\Phi_{v^{\prime}}$ for another vertex $v^{\prime} \in \Gamma$ with $h(v)=h\left(v^{\prime}\right)$. We say that a vertex $v$ is real if $\Phi_{v}$ is defined over $\mathbb{R}$.

Let $\mathbb{R} C_{\alpha}$ be a scaled sequence of type-I curves enhanced with a complex orientation, so that a component $S_{\alpha} \subset \mathbb{C} C_{\alpha} \backslash \mathbb{R} C_{\alpha}$ is fixed for all $\alpha$. Suppose that $\mathbb{C} C_{\alpha}$ has a phasetropical limit, and that the orientations of $\mathbb{R} C_{\alpha}$ agree with some complex orientations of the real part $\mathbb{R} \Phi(p)$ of the phases $\Phi(p)$. The quantum index of $\mathbb{R} C_{\alpha}$ is well defined if it has real or purely imaginary coordinate intersection. Similarly, the phase $\mathbb{R} \Phi_{v}$ of a real vertex $v$ of the tropical limit has a well-defined quantum index if $\sigma(E) \equiv 0(\bmod \pi)$ for any edge $E$ adjacent to $v$.

Proposition 6.14. For large $t_{\alpha}$ we have

$$
k\left(\mathbb{R} C_{\alpha}\right)=\sum_{v} k\left(\mathbb{R} \Phi_{v}\right),
$$

where the sum is taken over all real vertices, whenever all quantum indices in (6.20) are well defined.

Proof. Additivity of the quantum index with respect to the phases $\Phi_{v}$ follows from Theorem 3.1 through additivity of the degree of the map 2Arg restricted to $S \cap\left(\mathbb{C}^{\times}\right)^{2}$. Non-real vertices give zero contribution to $k\left(\mathbb{R} C_{\alpha}\right)$, as the signed area of the amoeba of the whole complex curve is zero. 
Proof of Theorem 5.9. Recall the definition of the (tropical) Block-Göttsche invariants (see [11]), which refine tropical enumerative invariants of [18]. Namely, with any 3 -valent (open) tropical immersed curve $h: \Gamma^{\circ} \rightarrow \mathbb{R}^{2}$ we may associate the Laurent polynomial

$$
n_{q}\left(h\left(\Gamma^{\circ}\right)\right)=\prod_{v} \frac{q^{m(v) / 2}-q^{-m(v) / 2}}{q^{1 / 2}-q^{-1 / 2}},
$$

where $v$ runs over all vertices $v \in \Gamma$ and $m(v)$ is the multiplicity of the vertex $v$. The genus of a (connected) tropical curve $\Gamma^{\circ}$ is the first Betti number of $\Gamma^{\circ}$. In particular, a rational tropical curve is a tree.

Let us fix a collection $\mu=\left\{\mu_{j}\right\}_{j=1}^{m}, m=\#\left(\partial \Delta \cap \mathbb{Z}^{2}\right)$, of generic real numbers subject to the condition $\sum_{j=1}^{m} \mu_{j}=0$. This means that $\mu_{j}, j=1, \ldots, m-1$, are chosen generically, and $\mu_{m}$ is determined from our condition.

If $h: \Gamma^{\circ} \rightarrow \mathbb{R}^{2}$ is a tropical curve with Newton polygon $\Delta$, then we number its leaves so that the first $m_{1}$ leaves are dual to the side $E \subset \partial \Delta$, the second $m_{2}$ to the side $E_{2} \subset \partial \Delta$, and so on with the last $m_{n}$ leaves dual to $E_{n}$. We say that $h: \Gamma \rightarrow \mathbb{R}^{2}$ passes through $\partial \mathbb{T} \Delta$ at the points determined by $\mu$ if the $j$ th unbounded edge of $\Gamma$ has momentum $\mu_{j}$. Note that a leaf $E \subset \Gamma^{\circ}$ must have momentum $\mu(E)$ if it passes through a point $p_{E}$ on the oriented line parallel to the vector $(d h) u(E)$ with momentum $\mu(E)$. Thus a generic choice of the momenta ensures that $h: \Gamma^{\circ} \rightarrow \mathbb{R}^{2}$ passes through a generic collection of $m-1$ points in $\mathbb{R}^{2}$. Thus, we have only finitely many rational tropical curves with Newton polygon $\Delta$ passing through $\partial \mathbb{T} \Delta$ at the points determined by $\mu$, and this is due to [18, Lemma 4.22] (as the number of combinatorial types of tropical curves with the given Newton polygon $\Delta$ is finite). By [18, Proposition 4.11], all these tropical curves are simple in the sense of [18, Definition 4.2].

The Block-Göttsche number associated with $\mu$ is

$$
N_{\Delta}^{\partial, \text { trop }}=N_{\Delta}^{\partial, \text { trop }}(\mu)=\sum_{h: \Gamma^{\circ} \rightarrow \mathbb{R}^{2}} n_{q}\left(h\left(\Gamma^{\circ}\right)\right),
$$

where the sum is taken over all $h: \Gamma^{\circ} \rightarrow \mathbb{R}^{2}$ passing through $\partial \mathbb{T} \Delta$ at the points determined by $\mu$. Independence of $N_{\Delta}^{\partial \text {,trop }}$ from $\mu$ can be proved in the same way as in [11]. Also, it follows from Theorem 5.5 once we prove the coincidence of $R_{\Delta}$ and $N_{\Delta}^{\partial \text {,trop }}(\mu)$.

A toric divisor $\mathbb{C} E_{j} \subset \mathbb{C} \Delta$ is the compactification of the torus $\mathbb{C}^{\times}$obtained by taking the quotient group of $\left(\mathbb{C}^{\times}\right)^{2}$ by the subgroup defined by the side $E_{j} \subset \Delta$. Thus, a configuration $\mathcal{P}=\left\{p_{j}\right\}_{j=1}^{m} \subset \partial \mathbb{C} \Delta$ is given by a collection of $m$ non-zero complex numbers, as well as an attribution of the points to the toric divisors. This collection is real if the corresponding numbers are real, and positive if these numbers are positive.

We let $\mathcal{P}^{t}=\left\{p_{1}^{t}, \ldots, p_{m}^{t}\right\} \subset \partial \mathbb{R} \Delta$ be the configuration of points with the same toric divisor attribution as $\mathcal{P}$, and given by the positive numbers $\left\{t^{2 \mu_{j}}\right\}, t>1$. As a consequence 
of [18, Proposition 8.7], the amoebas of rational complex curves with Newton polygon $\Delta$ passing through $\left(\mathrm{Sq}^{\Delta}\right)^{-1}\left(\mathcal{P}_{t}\right)$ converge when $t \rightarrow \infty$ to tropical curves passing through $\partial \mathbb{T} \Delta$ at the points determined by $\mu$. We have that [18, Proposition 8.23] determines the number of complex curves with amoeba in a small neighborhood of a rational tropical curve $h: \Gamma^{\circ} \rightarrow \mathbb{R}^{2}$ passing through any choice of points $\tilde{p}_{j}^{t} \in\left(\mathrm{Sq}^{\Delta}\right)^{-1}\left(p_{j}^{t}\right), j=1, \ldots, m-1$, for large $t$, while [18, Remark 8.25] determines the number of the corresponding real curves. E.g., if the weights of all edges of $\Gamma^{\circ}$ are odd, we have a single real curve for any choice of $\widetilde{\mathcal{P}}^{t}=\left\{\tilde{p}_{j}^{t}\right\}$. In general, some choices of $\widetilde{\mathcal{P}}_{t}$ may correspond to no real solutions, while others may correspond to multiple solutions. We claim that nevertheless there are $2^{m-1}$ different real curves whose amoeba is close to $h: \Gamma^{\circ} \rightarrow \mathbb{R}^{2}$ with the image under $\mathrm{Sq}^{\Delta}$ passing through $\mathcal{P}^{t}$ for large $t$. Thus, we have $2^{m}$ different oriented curves. We show this by induction on $m$ as follows.

If $\Gamma^{\circ}$ has a single vertex $v$ (so that $m=3$ ), then there are four different real rational phases $\Phi_{v}$ which differ by the deck transformations of the map $\mathrm{Sq}^{\Delta}$. Thus, we have eight different oriented real rational phases in this case. The positive logarithmic rotation number for half of them is positive, while for the other half is negative. Adding each new 3 -valent vertex $v^{\prime}$ to the tree $\Gamma$ doubles the number of oriented real phases, as there are two ways to attach the phase for $v^{\prime}$ : so that the logarithmic rotation number of the resulting real curve will increase by one, and so that it will decrease by one. Inductively, we get four real oriented curves for each of the $2^{m-2}$ possible sign distributions for the vertices of $\Gamma^{\circ}$.

For each vertex $v$, the real phase $\mathbb{R} \Phi_{v}$ is the image of a line by a multiplicative-linear map of determinant $m(v)$, by [18, Corollary 8.20]. Therefore $k\left(\mathbb{R} \Phi_{v}\right)= \pm \frac{1}{2} m(v)$, where the sign is determined by the degree of the logarithmic Gauss map. According to our sign convention (5.3), each oriented real curve comes with the sign equal to the number of negative vertices. Thus, by Proposition 6.14, the contribution of $h: \Gamma \rightarrow \mathbb{R}^{2}$ to $R_{\Delta}\left(\mathcal{P}^{t}\right)$ for large $t$ is $\prod_{v}\left(q^{m / 2}-q^{-m / 2}\right)$, which coincides with the numerator of the Block-Göttsche multiplicity (6.21).

Acknowledgment. The results of this paper were inspired by a discussion with Ilia Itenberg, Maxim Kontsevich and Ilia Zharkov on the Spring Equinox of 2015, before a traditional IHES volleyball game, as well as some discussions with Ivan Cherednik, Sergey Galkin and Yan Soibelman on some other occasions. In particular, an example of area computation for holomorphic disks with boundary in a union of two Lagrangian subvarieties explained by Maxim Kontsevich was especially helpful. The author would like to thank everybody for a fruitful exchange of ideas, as well as the IHES for its hospitality. The paper has tremendously benefited from many helpful remarks of the referee, to whom the author is sincerely thankful. 
Research was supported in part by the grants 141329, 159240, 159581 and the NCCR SwissMAP project of the Swiss National Science Foundation, as well as by the Chaire d'Excellence program of the Fondation Sciences Mathématiques de Paris.

\section{References}

[1] Block, F. \& Göttsche, L., Refined curve counting with tropical geometry. Compos. Math., 152 (2016), 115-151.

[2] Brugallé, E., Pseudoholomorphic simple Harnack curves. Enseign. Math., 61 (2015), $483-498$.

[3] Caporaso, L., Algebraic and tropical curves: comparing their moduli spaces, in Handbook of Moduli. Vol. I, Adv. Lectures Math., 24, pp. 119-160. Int. Press, Somerville, MA, 2013.

[4] Carnot, L. N. M., Géométrie de position. Imprimerie de Crapelet, Paris, 1803.

[5] Deligne, P., Le symbole modéré. Inst. Hautes Études Sci. Publ. Math., 73 (1991), 147181.

[6] Deligne, P. \& Mumford, D., The irreducibility of the space of curves of given genus. Inst. Hautes Études Sci. Publ. Math., 36 (1969), 75-109.

[7] Forsberg, M., Passare, M. \& Tsikh, A., Laurent determinants and arrangements of hyperplane amoebas. Adv. Math., 151 (2000), 45-70.

[8] Göttsche, L. \& Shende, V., Refined curve counting on complex surfaces. Geom. Topol., 18 (2014), 2245-2307.

[9] Griffiths, P. \& Harris, J., Principles of Algebraic Geometry. Wiley-Interscience, New York, 1978.

[10] Itenberg, I., Kharlamov, V. \& Shustin, E., Welschinger invariant and enumeration of real rational curves. Int. Math. Res. Not., 49 (2003), 2639-2653.

[11] ItenberG, I. \& Mikhalkin, G., On Block-Göttsche multiplicities for planar tropical curves. Int. Math. Res. Not., 23 (2013), 5289-5320.

[12] Kapranov, M. M., A characterization of $A$-discriminantal hypersurfaces in terms of the logarithmic Gauss map. Math. Ann., 290 (1991), 277-285.

[13] Kenyon, R., Okounkov, A. \& Sheffield, S., Dimers and amoebae. Ann. of Math., 163 (2006), 1019-1056.

[14] Kontsevich, M. \& Manin, Y., Gromov-Witten classes, quantum cohomology, and enumerative geometry. Comm. Math. Phys., 164 (1994), 525-562.

[15] Kontsevich, M. \& Solbelman, Y., Stability structures, motivic Donaldson-Thomas invariants and cluster transformations. Preprint, 2008. arXiv:0811.2435 [math.AG].

[16] Kushnirenko, A. G., Newton polyhedra and Bezout's theorem. Funkcional. Anal. $i$ Priložen., 10 (1976), 82-83 (Russian); English translation in Functional Anal. Appl., 10 (1976), 233-235.

[17] Mikhalkin, G., Real algebraic curves, the moment map and amoebas. Ann. of Math., 151 (2000), 309-326.

[18] - Enumerative tropical algebraic geometry in $\mathbb{R}^{2}$. J. Amer. Math. Soc., 18 (2005), 313377.

[19] - Tropical geometry and its applications, in International Congress of Mathematicians. Vol. II, pp. 827-852. Eur. Math. Soc., Zürich, 2006.

[20] - Amoebas of half-dimensional varieties, in Analysis Meets Geometry, The Mikael Passare Memorial Volume, pp. 349-359. Springer International Publishing, Cham, 2017. 
[21] Mikhalkin, G. \& Okounkov, A., Geometry of planar log-fronts. Mosc. Math. J., 7 (2007), 507-531, 575 .

[22] Mikhalkin, G. \& Rullgård, H., Amoebas of maximal area. Int. Math. Res. Not., 9 (2001), 441-451.

[23] Nekrasov, N. \& Okounkov, A., Membranes and sheaves. Algebr. Geom., 3 (2016), 320369.

[24] Passare, M., How to compute $\sum 1 / n^{2}$ by solving triangles. Amer. Math. Monthly, 115 (2008), 745-752.

[25] Passare, M. \& Rullgård, H., Amoebas, Monge-Ampère measures, and triangulations of the Newton polytope. Duke Math. J., 121 (2004), 481-507.

[26] Rohlin, V. A., Complex orientation of real algebraic curves. Funkcional. Anal. i Priložen., 8 (1974), 71-75 (Russian).

[27] Viro, O. Y., Achievements in the topology of real algebraic varieties in the last six years. Uspekhi Mat. Nauk, 41 (1986), 45-67, 240 (Russian); English translation in Russian Math. Surveys, 41 (1986), 55-82.

[28] Welschinger, J.-Y., Invariants of real symplectic 4-manifolds and lower bounds in real enumerative geometry. Invent. Math., 162 (2005), 195-234.

\author{
GRIGORY MIKHALKIN \\ Université de Genève \\ Mathématiques \\ Battelle Villa \\ 1227 Carouge \\ Switzerland \\ grigory.mikhalkin@unige.ch
}

Received January 4, 2016

Received in revised form November 22, 2017 